\title{
Physical Human-Robot Cooperation Based on Robust Motion Intention Estimation
}

\section{Konstantinos I. Alevizos, Charalampos P. Bechlioulis*๑ and Kostas J. Kyriakopoulos}

Control Systems Lab, School of Mechanical Engineering, National Technical University of Athens, 9 Heroon Polytechniou Street, Athens, 15780, Greece

(Accepted August 19, 2020)

\begin{abstract}
SUMMARY
Cooperative transportation by human and robotic coworkers constitutes a challenging research field that could lead to promising technological achievements. Toward this direction, the present work demonstrates that, under a leader-follower architecture, where the human determines the object's desired trajectory, complex cooperative object manipulation with minimal human effort may be achieved. More specifically, the robot estimates the object's desired motion via a prescribed performance estimation law that drives the estimation error to an arbitrarily small residual set. Subsequently, the motion intention estimation is utilized in the object dynamics to determine the interaction force between the human and the object. Human effort reduction is then achieved via an impedance control scheme that employs the aforementioned estimations. The feedback relies exclusively on the robot's force/torque, position as well as velocity measurements at its end effector, without incorporating any other information on the task. Moreover, an adaptive control scheme is adopted to relax the need for exact knowledge of the object dynamics. Finally, an extension for multiple robotic coworkers is studied and verified via simulation, while extensive experimental results for the single robot case clarify the proposed method and corroborate its efficiency.
\end{abstract}

KEYWORDS: RAAD2018; pHRI; Cooperative transportation; Prescribed performance; Impedance control.

\section{Introduction}

Although robots have been involved in industry for a long time, most cases refer to repetitive tasks implemented in isolated workplaces in the absence of human workers (see Fig. 1(a)). Nonetheless, as robots are advancing in both industry and everyday life, human-robot collaboration proves to be a vast and highly emerging field that aims at allowing humans and robots to safely share a common workspace while mutually assisting each other in meaningful tasks (see Fig. 1(b)). Among humanrobot collaboration, physical human-robot interaction (pHRI) could justifiably be characterized as a rather challenging research field especially for complex tasks previously performed by one or more humans. In particular, the present work focuses on cooperative object manipulation and envisages an architecture that prioritizes the benefits of available human cognitive skills while maximizing the autonomy of robotic partners. In this way, a combination of robot power with human planning and decision-making capabilities based solely on implicit communication provided by the physical interaction is expected to lead to a powerful and versatile technology for the industry.

* Corresponding author. E-mail: chmpechl@mail.ntua.gr 

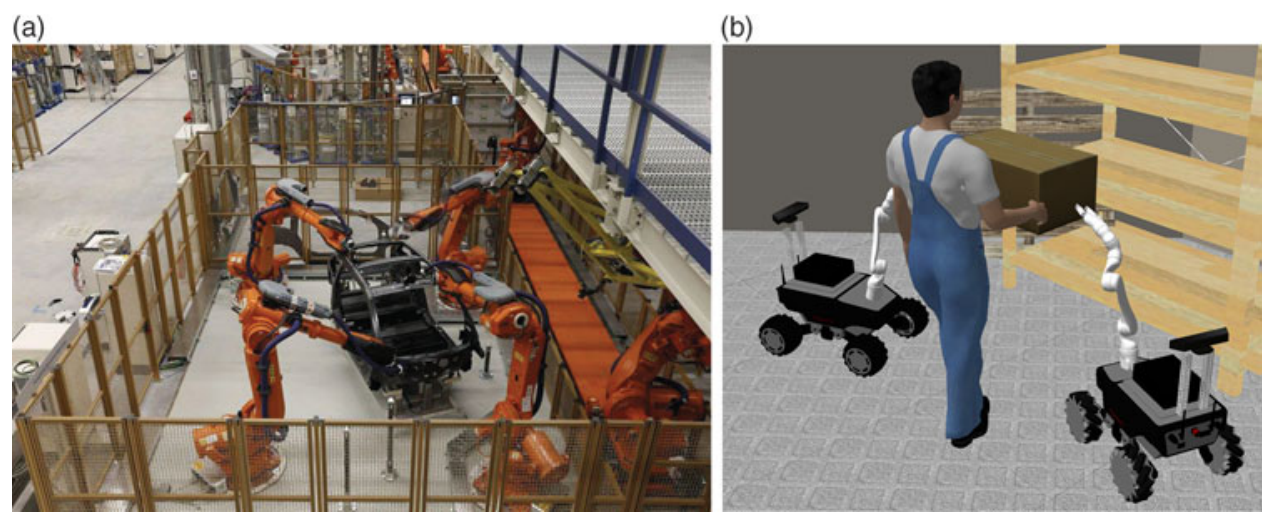

Fig. 1. (a) Robots in industry. (b) Human-robot cooperative transportation task.

Related research has been conducted with early results pertaining to passive robotic assistants. As shown in refs. [1,2], such systems cooperate well with humans through physical interaction and may be useful in the transportation of bulky objects, although they lack the ability to reduce human effort by actively contributing to the transportation task. On the contrary, cooperative human-robot object manipulation faces various technical issues toward the integration of a proactive system that resembles the execution of cooperative tasks explicitly by humans. In ref. [3], confronting the concept of role assignment, novel strategies have been developed for effort sharing. Although these strategies can be very helpful in terms of effort reduction, the requirement for predefined globally known task trajectory makes them applicable mostly for repetitive tasks in well-structured environments.

Alternatively, control schemes that do not assume a priori knowledge of the desired trajectory have been developed to provide more autonomous and reactive solutions. In these works, the desired trajectory that is explicitly determined by humans is estimated based on the system's sensing ability. Usually, a force control scheme is designed based on these estimates to impose the desired characteristics on the system. In ref. [4], for a simple transportation task with known starting and ending points, Kalman filtering is used to estimate the parameters of the minimum jerk profile. Likewise, in ref. [5], non-linear least squares were employed in a similar task. Therefore, the results were fed into an admittance and impedance control scheme. Estimations based on minimum-jerk profile showed interesting results, but these strategies require a known goal position. However, profiles that correspond to more complex tasks than point-to-point motion have not been considered. An EKF was also used in ref. [6] for prediction of the human's desired trajectory and was effectively combined with a reactive reinforcement learning controller to develop a behavior-based gain controller that relies on prediction confidence levels for a cooperative lifting task. In refs. [7-9] based on impedance control, a controller is defined for co-manipulation of unknown weight parts. The first uses only force, position and velocity measurements, while the rest use various machine learning techniques to optimize the control parameters to reduce human effort. Another approach for human motion intention estimation is presented in ref. [10], where a neural network was trained to predict human motion intention using the position, velocity and force measurements as inputs. The predicted trajectory was then provided to an adaptive impedance controller. To the best of our knowledge, experimental verification of the aforementioned works was restricted to either $1 \mathrm{DoF}$ experiments or plain tasks in higher dimensions.

Other modern approaches rely on task-specific learning and programming-by-demonstration techniques. Plenty of these approaches also provide experimental evaluation for complex transportation or manipulation tasks. In ref. [11], a statistical model, based on Gaussian mixture models, was trained in a pure leader-follower role distribution after a set of demonstrations. The task, which was an object lifting scenario, was autonomously reproduced using Gaussian mixture regression. Demonstrations were also employed in ref. [12] to reproduce a task-parametrized formulation of a Gaussian mixture model, from which the desired trajectory was extracted. Moreover, based on the same statistical model, optimal controllers have been designed with respect to the variance of the robot's state measurements during the demonstrated task. In ref. [13], an experience-based scheme was proposed, where the robot was in compliant control and detected/classified, based on hidden Markov models, interaction patterns generated during transportation tasks. Human cognitive skills were further employed to rate the recognized task segments when necessary. The robot after some repetitions 
was able to predict the desired trajectory and implement it through an admittance scheme. Although these works have shown remarkable results, the non-negligible overhead of training renders them inconvenient for industrial applications.

This paper addresses the motion intention estimation problem via a robust prescribed performance estimation law that drives the error between the actual and the desired trajectory estimation to an arbitrarily small residual set. The proposed method is based only on position, velocity and interaction force/torque measurements at the robot end effector. In this respect, the interaction force/torque between the human and the object, which is unknown and cannot thus be employed in the control design, is determined by the object's dynamics and the estimation of the desired trajectory. Subsequently, based on the aforementioned motion intention estimation, an impedance control scheme is designed that leads in significant human's effort reduction through the impedance specifications of the controller. Moreover, the control scheme is extended for the non-trivial case of uncertainties in object dynamics. An adaptive scheme is proposed to overcome the unknown object dynamics contributing to the system's autonomy and robustness since only a rough estimate of the object's parameters is required. A cooperative transportation task between a human leader and multiple robotic agents is also examined. In the multiple agent scenario, alongside the decentralized estimation law, an impedance control scheme was developed to augment further the human capability to handle heavy and bulky objects, necessitating however for lean inter-robot communication. In this paper, we demonstrate that we are able to achieve cooperative object transportation for challenging $6 \mathrm{DoF}$ trajectories and execute complex transportation tasks without resorting to learning techniques. In conclusion, the main contributions of the present work are:

- a robust estimation algorithm that converges even though the desired object's acceleration profile is nonzero (i.e., the arbitrary object's desired trajectory based on human motion planning, as long as it is bounded and smooth)

- the successful integration of the estimation with an impedance control scheme in order to reduce the required human effort for a collaborative task.

- reinforcement of the closed-loop robustness by incorporating parametric uncertainty in the object dynamics.

The rest of the manuscript is organized as follows. In Section 2, preliminaries related to the kinematics and dynamics of the system components are provided. Section 3 presents the problem statement and the proposed strategy in detail. Experimental results and simulation studies are given in Sections 4 and 5, respectively. Finally, Section 6 concludes the paper.

\section{Preliminaries}

In this paper, a human-robot cooperative system is investigated that involves a human and one or more robotic manipulators in a leader-follower architecture, handling a rigidly grasped object. It is assumed that each robotic manipulator has at least $6 \mathrm{DoFs}$, is fully actuated and is equipped with a force/torque sensing handle at its end effector. Although the geometric and inertial parameters of the grasped object are initially considered known, such assumption is relaxed later by employing an appropriately designed adaptive scheme.

\subsection{Kinematics}

Let us denote the frames $\Sigma_{k}, k \in\left\{l, f_{1}, \ldots, f_{N}\right\}$, where the subscripts $l, f$ stand for leader and follower, respectively and $N$ is the number of manipulators attached to the object. The origin and the orientation of each frame $\Sigma_{k}$ with respect to an inertial frame $\left\{\Sigma_{I}\right\}^{1}$ are given by $\boldsymbol{p}_{k} \in \mathfrak{R}^{3}$ and $\boldsymbol{R}_{k} \in S O(3)$. We denote the angular velocity vector as $\boldsymbol{\omega}_{k} \in \mathfrak{R}^{3}$ and the generalized velocity vector as $\boldsymbol{v}_{k}=\left[\dot{\boldsymbol{p}}_{k}^{T}, \boldsymbol{\omega}_{k}^{T}\right]^{T}$. We denote also the unit quaternion derived from $\boldsymbol{R}_{k}$ as $\mathcal{Q}_{k}=\left\{\eta_{k}, \boldsymbol{\epsilon}_{k}\right\} \in S^{3} \subset \mathfrak{R}^{4}$. For a unit quaternion $\mathcal{Q}_{k}=\{\eta, \boldsymbol{\epsilon}\}$, it holds that $\eta=\cos \frac{\theta}{2}, \boldsymbol{\epsilon}=\sin \frac{\theta}{2} \boldsymbol{r}$ are the scalar and vector part, respectively, and they are constrained by

$$
\eta^{2}+\boldsymbol{\epsilon}^{T} \boldsymbol{\epsilon}=1
$$

${ }^{1}$ The frame with respect to which a quantity is expressed is denoted by a right superscript that is, $\boldsymbol{p}_{k}^{I}$. The superscript $I$ is dropped for simplicity when the quantity is expressed in $\Sigma_{I}$ that is, $\boldsymbol{p}_{k}$. The frame $\Sigma_{I}$ is also referred as base frame. 
The relation between the time derivatives of $\dot{\eta}, \dot{\boldsymbol{\epsilon}}$ and the angular velocity $\omega$ is described by

$$
\begin{aligned}
& \dot{\eta}=-\frac{1}{2} \boldsymbol{\epsilon}^{T} \boldsymbol{\omega} \\
& \dot{\boldsymbol{\epsilon}}=\frac{1}{2} \boldsymbol{E}(\eta, \boldsymbol{\epsilon}) \boldsymbol{\omega}
\end{aligned}
$$

with

$$
\boldsymbol{E}(\eta, \boldsymbol{\epsilon})=\eta \boldsymbol{I}-S(\boldsymbol{\epsilon})
$$

where $S($.) denotes the skew symmetric matrix operator performing the cross product. Similarly, let frame $\Sigma_{o}$ be attached to the object's center of mass with the corresponding quantities denoted by $\boldsymbol{p}_{o} \in \mathfrak{R}^{3}, \boldsymbol{R}_{o} \in S O(3), \mathcal{Q}_{o}=\left\{\eta_{o}, \boldsymbol{\epsilon}_{o}\right\} \in S^{3} \subset \mathfrak{R}^{4}, \boldsymbol{v}_{o}=\left[\dot{\boldsymbol{p}}_{o}^{T}, \boldsymbol{\omega}_{o}^{T}\right]^{T} \in \mathfrak{R}^{6}$. Notice that the frame $\Sigma_{o}$ can be attached to the object's center of mass only when it is considered known. If such information is not available, then $\Sigma_{o}$ can be chosen as an arbitrary frame on the object. In this case, a preferable choice for the origin of $\Sigma_{o}$ could be the mean vector of all grasping points, that is, $\frac{\sum_{i=1}^{N} p_{f_{i}}}{N}$.

Moreover, let $\boldsymbol{q}_{f_{i}}, i \in\{1, \ldots, N\}$ be the joint space variables of the i-th manipulator and $\boldsymbol{J}_{f_{i}}\left(\boldsymbol{q}_{f_{i}}\right)$ be the geometric Jacobian matrix such as

$$
\boldsymbol{v}_{f_{i}}=\boldsymbol{J}_{f_{i}}\left(\boldsymbol{q}_{f_{i}}\right) \dot{\boldsymbol{q}}_{f_{i}}
$$

Following the virtual stick ${ }^{14}$ convention, we can augment each manipulator by a constant translation and rotation such that each of the manipulator's virtual end effector coincides with the origin $\Sigma_{o}$. The same convention holds for the human arm. Assuming that the contacts are rigid, the following kinematic constraints hold:

$$
\begin{aligned}
& \boldsymbol{p}_{k}=\boldsymbol{p}_{o}-\boldsymbol{r}_{k} \\
& \boldsymbol{R}_{k}=\boldsymbol{R}_{o} \boldsymbol{R}_{k}^{o}
\end{aligned}
$$

where $\boldsymbol{r}_{k} \in \mathfrak{R}^{3}$ denotes the virtual stick from the k-th end effector (or the human hand) to the origin of $\Sigma_{o}$, while $\boldsymbol{R}_{k}^{o}$ refers to the relative orientation between $\Sigma_{o}$ and $\Sigma_{k}$. Differentiating (6) and (7) and using the relation $\dot{\boldsymbol{R}}_{k}=\boldsymbol{S}\left(\boldsymbol{\omega}_{k}\right) \boldsymbol{R}_{k}$, we can derive the following:

$$
\begin{aligned}
& \dot{\boldsymbol{p}}_{k}=\dot{\boldsymbol{p}}_{o}-\boldsymbol{r}_{k} \times \boldsymbol{\omega}_{o} \\
& \boldsymbol{\omega}_{k}=\boldsymbol{\omega}_{o}
\end{aligned}
$$

or in compact matrix form

$$
\boldsymbol{v}_{k}=\boldsymbol{J}_{o k} \boldsymbol{v}_{o}=\left[\begin{array}{cc}
\boldsymbol{I}_{3 \times 3} & -\boldsymbol{S}\left(\boldsymbol{r}_{k}\right) \\
\mathbf{0}_{3 \times 3} & \boldsymbol{I}_{3 \times 3}
\end{array}\right] \boldsymbol{v}_{o}
$$

where $\boldsymbol{J}_{o k}$ is the Jacobian from the end effector (or the human hand) to $\Sigma_{o}$. Notice that since the end effector and the object are rigidly connected, the aforementioned Jacobian is always full rank and hence a well-defined inverse $\boldsymbol{J}_{o k}^{-1}$ exists. Finally, differentiating (10) with respect to time, we also establish the generalized acceleration relation:

$$
\dot{\boldsymbol{v}}_{k}=\dot{\boldsymbol{J}}_{o k} \boldsymbol{v}_{o}+\boldsymbol{J}_{o k} \dot{\boldsymbol{v}}_{o}
$$

2.2. Manipulator dynamics

The dynamics of the manipulator can be written in the form of:

$$
\boldsymbol{M}_{r_{i}}\left(\boldsymbol{q}_{f_{i}}\right) \ddot{\boldsymbol{q}}_{f_{i}}+\boldsymbol{C}_{r_{i}}\left(\dot{\boldsymbol{q}}_{f_{i}}, \boldsymbol{q}_{f_{i}}\right) \dot{\boldsymbol{q}}_{f_{i}}+\boldsymbol{G}_{r_{i}}\left(\boldsymbol{q}_{f_{i}}\right)=\boldsymbol{\tau}_{f_{i}}+\boldsymbol{J}_{f_{i}}^{T}\left(\boldsymbol{q}_{f_{i}}\right) \boldsymbol{h}_{f_{i}}, i \in\{1, \ldots, N\}
$$

where $\boldsymbol{\tau}_{f i} \in \Re^{m}$ is the torque applied to each actuator, $\boldsymbol{M}_{r_{i}}\left(\boldsymbol{q}_{f_{i}}\right) \in \mathfrak{R}^{m \times m}$ is the symmetric, positive definite mass matrix, $\boldsymbol{C}_{r_{i}}\left(\dot{\boldsymbol{q}}_{f_{i}}, \boldsymbol{q}_{f_{i}}\right) \in \Re^{m \times m}$ is the Coriolis/centrifugal matrix, $\boldsymbol{G}_{r_{i}}\left(\boldsymbol{q}_{f_{i}}\right) \in \Re^{m}$ represents the 
gravitational forces and $\boldsymbol{h}_{f_{i}} \in \Re^{m}$ denotes the forces exerted by the environment on the end effector. The dynamic model of the robot, in terms of task space coordinates, is described by:

$$
\boldsymbol{M}_{f_{i}}\left(\boldsymbol{q}_{f_{i}}\right) \dot{\boldsymbol{v}}_{f_{i}}+\boldsymbol{C}_{f_{i}}\left(\dot{\boldsymbol{q}}_{f_{i}}, \boldsymbol{q}_{f_{i}}\right) \boldsymbol{v}_{f_{i}}+\boldsymbol{G}_{f_{i}}\left(\boldsymbol{q}_{f_{i}}\right)=\boldsymbol{u}_{f_{i}}+\boldsymbol{h}_{f_{i}}
$$

where

$$
\begin{aligned}
\boldsymbol{M}_{f_{i}}\left(\boldsymbol{q}_{f_{i}}\right) & \triangleq \boldsymbol{J}_{f_{i}}^{-T} \boldsymbol{M}_{r_{i}}\left(\boldsymbol{q}_{f_{i}}\right) \boldsymbol{J}_{f_{i}}^{-1} \\
\boldsymbol{C}_{f_{i}}\left(\dot{\boldsymbol{q}}_{f_{i}}, \boldsymbol{q}_{f_{i}}\right) & \triangleq \boldsymbol{J}_{f_{i}}^{-T}\left(\boldsymbol{C}_{r_{i}}\left(\dot{\boldsymbol{q}}_{f_{i}}, \boldsymbol{q}_{f_{i}}\right) \boldsymbol{J}_{f_{i}}^{-1}+\boldsymbol{M}_{r_{i}}\left(\boldsymbol{q}_{f_{i}}\right) \dot{\boldsymbol{J}}_{f_{i}} \boldsymbol{J}_{f_{i}}^{-1}\right) \\
\boldsymbol{G}_{f_{i}}\left(\boldsymbol{q}_{f_{i}}\right) & \triangleq \boldsymbol{J}_{f_{i}}^{-T} \boldsymbol{G}_{r_{i}}\left(\boldsymbol{q}_{f_{i}}\right)
\end{aligned}
$$

and $\boldsymbol{u}_{f_{i}}$ denotes the task space input wrench. The relation between the joint torques $\boldsymbol{\tau}_{f_{i}}$ and the task space wrench is given by $\boldsymbol{\tau}_{f_{i}}=\overline{\boldsymbol{J}}_{f_{i}}^{T} \boldsymbol{u}_{f_{i}}+\left(\boldsymbol{I}-\boldsymbol{J}_{f_{i}}^{T} \overline{\boldsymbol{J}}_{f_{i}}^{T}\right) \boldsymbol{\tau}_{i n_{i}}$, where $\overline{\boldsymbol{J}}_{f_{i}}$ is the generalized inverse that is consistent with the equations of motion of the manipulator and its end effector. ${ }^{15}$ The vector $\boldsymbol{\tau}_{i n_{i}}$ does not contribute to the end effector's wrench and can be regulated independently to achieve secondary goals (e.g., manipulability increase or collision avoidance for the links). Additionally, invoking the kinematic relations (6)-(11), we may express the aforementioned dynamic model (13) with respect to the object's coordinates as follows:

$$
\boldsymbol{M}_{o f_{i}}\left(\boldsymbol{q}_{f_{i}}\right) \dot{\boldsymbol{v}}_{o}+\boldsymbol{C}_{o f_{i}}\left(\dot{\boldsymbol{q}}_{f_{i}}, \boldsymbol{q}_{f_{i}}\right) \boldsymbol{v}_{o}+\boldsymbol{G}_{o f_{i}}\left(\boldsymbol{q}_{f}\right)=\boldsymbol{J}_{o f_{i}}^{T} \boldsymbol{u}_{f_{i}}+\boldsymbol{J}_{o f_{i}}^{T} \boldsymbol{h}_{f_{i}}
$$

where

$$
\begin{aligned}
\boldsymbol{M}_{o f_{i}}\left(\boldsymbol{q}_{f_{i}}\right) & \triangleq \boldsymbol{J}_{o f_{i}}^{T} \boldsymbol{M}_{f_{i}}\left(\boldsymbol{q}_{f_{i}}\right) \boldsymbol{J}_{o f_{i}} \\
\boldsymbol{C}_{o f_{i}}\left(\dot{\boldsymbol{q}}_{f_{i}}, \boldsymbol{q}_{f_{i}}\right) & \triangleq \boldsymbol{J}_{o f_{i}}^{T}\left(\boldsymbol{C}_{f_{i}}\left(\dot{\boldsymbol{q}}_{f_{i}}, \boldsymbol{q}_{f_{i}}\right) \boldsymbol{J}_{o f_{i}}+\boldsymbol{M}_{f_{i}}\left(\boldsymbol{q}_{f_{i}}\right) \dot{\boldsymbol{J}}_{o f_{i}}\right) \\
\boldsymbol{G}_{o f_{i}}\left(\boldsymbol{q}_{f_{i}}\right) & \triangleq \boldsymbol{J}_{o f_{i}}^{T} \boldsymbol{G}_{f_{i}}\left(\boldsymbol{q}_{f_{i}}\right)
\end{aligned}
$$

Finally, notice that the overall dynamics can be formulated in compact form as follows:

$$
\boldsymbol{M}_{O F} \dot{\boldsymbol{V}}_{o}+\boldsymbol{C}_{O F} \boldsymbol{V}_{o}+\boldsymbol{G}_{O F}=\boldsymbol{J}_{O F}^{T}\left(\boldsymbol{U}+\boldsymbol{H}_{f}\right)
$$

where

$$
\begin{aligned}
& \boldsymbol{M}_{O F} \triangleq \operatorname{blockdiag}\left\{\boldsymbol{M}_{o f_{1}}, \ldots, \boldsymbol{M}_{o f_{N}}\right\} \\
& \boldsymbol{C}_{O F} \triangleq \operatorname{blockdiag}\left\{\boldsymbol{C}_{o f_{1}}, \ldots, \boldsymbol{C}_{o f_{N}}\right\} \\
& \boldsymbol{J}_{O F} \triangleq \operatorname{blockdiag}\left\{\boldsymbol{J}_{o f_{1}}, \ldots, \boldsymbol{J}_{o f_{N}}\right\} \\
& \boldsymbol{G}_{O F} \triangleq\left[\boldsymbol{G}_{o f_{1}}^{T}, \ldots, \boldsymbol{G}_{o f_{N}}^{T}\right]^{T} \\
& \boldsymbol{H}_{f} \triangleq\left[\boldsymbol{h}_{f_{1}}^{T}, \ldots, \boldsymbol{h}_{f_{N}}^{T}\right]^{T} \\
& \boldsymbol{U} \triangleq\left[\boldsymbol{u}_{f_{1}}^{T}, \ldots, \boldsymbol{u}_{f_{N}}^{T}\right]^{T} \\
& \boldsymbol{V}_{o} \triangleq\left[\boldsymbol{v}_{o}^{T}, \ldots, \boldsymbol{v}_{o}^{T}\right]^{T}
\end{aligned}
$$

\subsection{Human limb dynamics}

A generic model that describes an approximation on the dynamics of a human limb motion involves a mass-damper-spring property, as in ref. [16]. However, our approach does not require any knowledge on the dynamical model of the human limb; thus, the following expression is given only to clarifythe 
notation. Similarly to the robot's case, we may express the aforementioned dynamical model with respect to the object's coordinates, invoking the kinematic relations (6)-(11), as follows:

$$
g\left(\Delta \dot{\boldsymbol{v}}_{d_{l} o}^{o}, \Delta \boldsymbol{v}_{d_{l} o}^{o}, \Delta \boldsymbol{x}_{d_{l} o}^{o}\right)=\boldsymbol{h}_{l o}^{o}
$$

where $g\left(\right.$.) represents human arm dynamics. The quantities $\boldsymbol{p}_{d_{l}} \in \mathfrak{R}^{3}, \boldsymbol{R}_{d_{l}} \in S O(3), \mathcal{Q}_{d_{l}}=\left\{\eta_{d_{l}}, \boldsymbol{\epsilon}_{d_{l}}\right\}$, $\boldsymbol{v}_{d_{l}}=\left[\dot{\boldsymbol{p}}_{d_{l}}^{T}, \boldsymbol{\omega}_{d_{l}}^{T}\right]^{T} \in \Re^{6}$ describe the desired trajectory planned by the central nervous system of the human leader with respect to the base frame. The dynamical model depends on the errors $\Delta \dot{\boldsymbol{v}}_{d_{1} o}^{o}=\dot{\boldsymbol{v}}_{d_{l}}^{o}-\dot{\boldsymbol{v}}_{o}^{o}, \Delta \boldsymbol{v}_{d_{l} o}^{o}=\boldsymbol{v}_{d_{l}}^{o}-\boldsymbol{v}_{o}^{o}, \Delta \boldsymbol{x}_{d_{l} o}^{o}=\left[\left(\boldsymbol{p}_{d_{l}}^{o}-\boldsymbol{p}_{o}^{o}\right)^{T}, \boldsymbol{\epsilon}_{d_{l} o}^{o T}\right]^{T}$ between the human's desired trajectory and the actual trajectory of the object, where $\epsilon_{d_{l} o}^{o}$ denotes the vector part of the quaternion $\mathcal{Q}_{d_{l} o}=\left\{\eta_{d_{l} o}, \boldsymbol{\epsilon}_{d_{l} o}\right\}$ that derives from their relative orientation. Finally, $\boldsymbol{h}_{l o}^{o}=\left[\boldsymbol{f}_{l o}^{o T}, \boldsymbol{\mu}_{l o}^{o T}\right]^{T}=\boldsymbol{J}_{o l}^{T} \boldsymbol{h}_{l}^{o}$ represents the forces and torques exerted on the human from the interaction with the object, and $\boldsymbol{J}_{o l}$ is the Jacobian from the human's hand to the end of the virtual stick.

\subsection{Object dynamics}

Under the hypothesis that the commonly grasped object is rigid (i.e., no deformations take place under the action of the applied forces by the human and the robots) and the grasp is rigid too (i.e., no relative motion between the grippers and the object takes place during the interaction), the following rigid body dynamics hold:

$$
\boldsymbol{M}_{o} \dot{\boldsymbol{v}}_{o}^{o}+\boldsymbol{C}_{o}\left(\boldsymbol{v}_{o}^{o}\right) \boldsymbol{v}_{o}^{o}+\boldsymbol{G}_{o}=-\boldsymbol{h}_{f o}^{o}-\boldsymbol{h}_{l o}^{o}
$$

where

$$
\boldsymbol{M}_{o} \triangleq\left[\begin{array}{cc}
m_{o} \boldsymbol{I}_{3 \times 3} & \mathbf{0}_{3 \times 3} \\
\mathbf{0}_{3 \times 3} & \boldsymbol{I}_{o}
\end{array}\right], \boldsymbol{C}_{o} \triangleq\left[\begin{array}{cc}
m_{o} \boldsymbol{S}\left(\boldsymbol{\omega}_{o}^{o}\right) & \mathbf{0}_{3 \times 3} \\
\mathbf{0}_{3 \times 3} & \boldsymbol{S}\left(\boldsymbol{\omega}_{o}^{o}\right) \boldsymbol{I}_{o}
\end{array}\right], \boldsymbol{G}_{o} \triangleq\left[\begin{array}{c}
m_{o} \boldsymbol{g}^{o} \\
\mathbf{0}_{3}
\end{array}\right]
$$

model inertial, Coriolis/centrifugal and gravitational effects, respectively, $\mathbf{0}_{3 \times 3} \in \mathfrak{R}^{3 \times 3}$ and $\boldsymbol{I}_{3 \times 3}$ are the zero and identity matrices and $m_{o}, \boldsymbol{I}_{o}$ stand for the mass and inertia tensor of the object. The right hand side of the equation denotes the generalized force exerted on the object by the human and the manipulators with $\boldsymbol{h}_{f o}^{o} \in \Re^{6}$ denoting the wrench exerted by the manipulators on frame $\Sigma_{o}$, such that

$$
\boldsymbol{h}_{f o}^{o}=\boldsymbol{G H}_{f}^{o}
$$

with

$$
\boldsymbol{G}=\left[\begin{array}{llll}
\boldsymbol{J}_{o f_{1}}^{T} & \boldsymbol{J}_{o f_{2}}^{T} & \cdots & \boldsymbol{J}_{o f_{N}}^{T}
\end{array}\right]
$$

denoting the grasp matrix of the $N$ robots and

$$
\boldsymbol{H}_{f}^{o} \triangleq\left[\boldsymbol{h}_{f_{1}}^{o T}, \ldots, \boldsymbol{h}_{f_{N}^{o}}^{T}\right]^{T}
$$

Under the assumption that the system is in a configuration that $\boldsymbol{G}$ is full row rank, from the inverse solution of (19), we obtain:

$$
\boldsymbol{H}_{f}^{o}=\overline{\boldsymbol{G}} \boldsymbol{h}_{f o}^{o}+\boldsymbol{V} \boldsymbol{h}_{I}^{o}=\overline{\boldsymbol{G}} \boldsymbol{G} \boldsymbol{H}_{f}^{o}+\boldsymbol{V} \boldsymbol{h}_{I}^{o}
$$

where $\overline{\boldsymbol{G}}$ is a pseudoinverse of $\boldsymbol{G}$ (for more details refer to ref. [17]) and $\boldsymbol{V}$ is the full column rank matrix that spans the null space of $\boldsymbol{G}$. Notice that $\boldsymbol{h}_{I} \in \mathfrak{R}^{6}$ which is also referred as internal force can be any vector that lies in the null-space of $\boldsymbol{G}$, and thus results in zero wrench on the object. Hence, the vector $\overline{\boldsymbol{G}} \boldsymbol{h}_{f o}^{o}$ represents the wrenches that contribute to the object motion, while the vector $\boldsymbol{V} \boldsymbol{h}_{I}^{o}$ represents the internal forces that contribute to pure mechanical stresses.

Finally, considering the case where no information about the object's dynamics and consequently its center of mass is provided, $\Sigma_{o}$ can be set to an arbitrary point on the object, to which all the virtual links of the robotic agents end up. Then, we can express the object dynamics in linear parameterizable form as in ref. [18] with respect to $\Sigma_{o}$ as:

$$
\boldsymbol{Y}\left(\boldsymbol{v}_{o}^{o}, \dot{\boldsymbol{v}}_{o}^{o}\right) \boldsymbol{\theta}=-\boldsymbol{h}_{f o}^{o}-\boldsymbol{h}_{l o}^{o}
$$




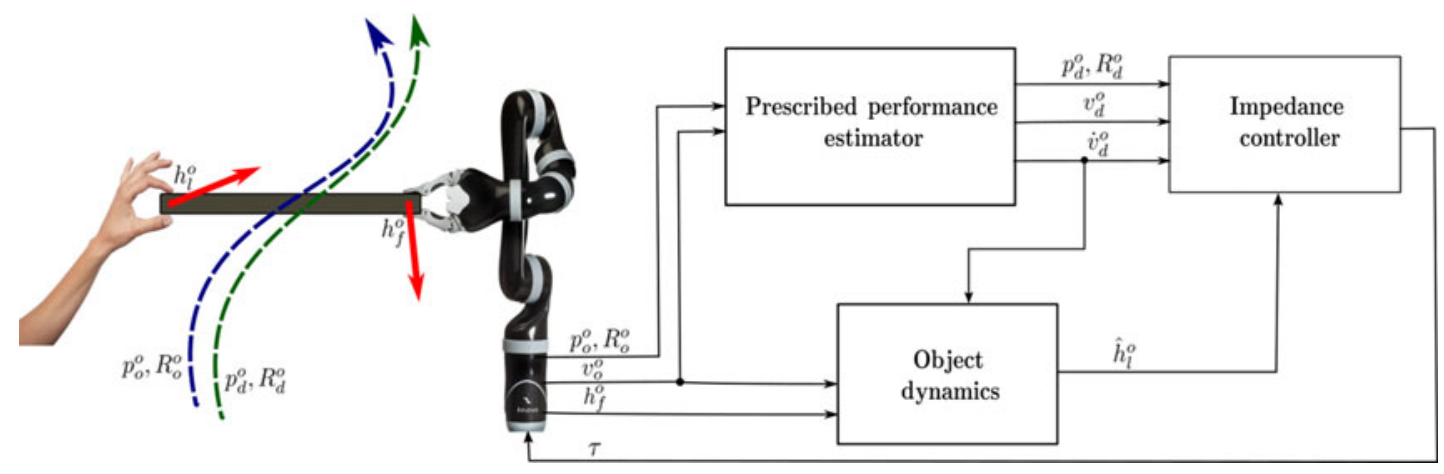

Fig. 2. Control diagram of the proposed method.

where

$$
\boldsymbol{Y}\left(\boldsymbol{v}_{o}^{o}, \dot{\boldsymbol{v}}_{o}^{o}\right)=\left[\boldsymbol{Y}_{p}\left(\boldsymbol{v}_{o}^{o}, \dot{\boldsymbol{v}}_{o}^{o}\right)^{T}, \boldsymbol{Y}_{r}\left(\boldsymbol{v}_{o}^{o}, \dot{\boldsymbol{v}}_{o}^{o}\right)^{T}\right]^{T} \in \Re^{6 \times 10}
$$

with $\boldsymbol{Y}_{p}, \boldsymbol{Y}_{r} \in \mathfrak{R}^{3 \times 10}$ and $\boldsymbol{\theta} \in \mathfrak{R}^{10}$ the parameter vector.

\section{Problem Statement and Control Methodology}

In a human-robot collaboration task, the human should lead by simply applying forces/torques to the object and the robot should "actively" follow by undertaking the object's load, thus reducing the required human effort. In contrast with a conventional robot task, where the desired trajectory is $a$ priori known, in a collaborative task, the desired trajectory is exclusively determined by the human and thus cannot be considered in the robot control design. Hence, the design objectives mainly consist of building a motion intention estimation law and subsequently an impedance scheme, to which the estimation serves as a reference. Specifically, the control scheme architecture can be interpreted as follows. In time step $i$, the human leader exerts an external wrench on the object. The external wrench changes the object's position/orientation from $\boldsymbol{p}_{o_{i-1}}^{o}, \boldsymbol{R}_{o_{i-1}}^{o}$ to $\boldsymbol{p}_{o_{i}}^{o}, \boldsymbol{R}_{o_{i}}^{o}$, similarly for velocity and acceleration. The wrench exerted on the human leader by the object is then defined by the desired impedance, whose setpoint is set in time step $i$-1 by the estimation law as $\boldsymbol{p}_{d_{i-1}}^{o}, \boldsymbol{R}_{d_{i-1}}^{o}, \dot{\boldsymbol{p}}_{d_{i-1}}^{o}, \boldsymbol{\omega}_{d_{i-1}}^{o}, \ddot{\boldsymbol{p}}_{d_{i-1}}^{o}$ and $\dot{\boldsymbol{\omega}}_{d_{i-1}}^{o}$. At the same time, the position/orientation, velocity measurements form the errors that are employed to estimate $\boldsymbol{p}_{d_{i}}^{o}, \boldsymbol{R}_{d_{i}}^{o}, \dot{\boldsymbol{p}}_{d_{i}}^{o}, \omega_{d_{i}}^{o}, \ddot{\boldsymbol{p}}_{d_{i}}^{o}$ and $\dot{\boldsymbol{\omega}}_{d_{i}}^{o}$ (initially, in $i=0$, the desired and actual frames are considered identical). Thus, if the estimation is valid, then we will be able to keep the stiffness of the desired impedance to a minimum, impose low damped behavior on the system and estimate the leader's wrench from the $\mathrm{f} / \mathrm{t}$ measurement in order to reduce human effort during the transportation task. An abstraction of the proposed methodology is depicted in Fig. 2.

\subsection{Motion intention estimation law}

In order to achieve the desired impedance behavior, the estimation law should not only estimate the object's desired trajectory profile $\boldsymbol{p}_{d}^{o}, \boldsymbol{R}_{d}^{o}, \boldsymbol{v}_{d}^{o}, \boldsymbol{\omega}_{d}^{o}$ but also compensate for acceleration residuals, since acceleration measurements are not available. In this respect, we relax the specification on asymptotic estimation by adopting a robust prescribed performance estimator that guarantees ultimate boundedness of the estimation errors $\boldsymbol{e}_{p}(t)=\Delta \boldsymbol{p}_{d o}^{o}=\boldsymbol{p}_{d}^{o}(t)-\boldsymbol{p}_{o}^{o}(t), \boldsymbol{e}_{r}(t)=\boldsymbol{\epsilon}_{d o}^{o}$ for position and orientation, respectively ${ }^{2}$. The mathematical representation of prescribed performance for each element of $\boldsymbol{e}(t) \triangleq\left[\boldsymbol{e}_{p}^{T}(t), \boldsymbol{e}_{r}^{T}(t)\right]^{T}=\left[e_{1}(t), \ldots, e_{6}(t)\right]^{T}$ is given by the following inequalities:

$$
-\rho_{j}(t)<e_{j}(t)<\rho_{j}(t), \forall t \geq 0, j \in\{1, \ldots, 6\}
$$

where $\rho_{j}(t)$ denotes the corresponding performance function that encapsulates the desired transient and steady-state performance specifications (e.g., convergence rate, maximum steady-state error). A candidate exponential performance function may be defined as:

$$
\rho_{j}(t)=\left(\rho_{j, 0}-\rho_{j, \infty}\right) e^{-s_{j} t}+\rho_{j, \infty}
$$

\footnotetext{
${ }^{2}$ Notice that the $i$ subscript used to discriminate between followers is dropped in this paragraph for simplicity.
} 
where the constant $s_{j}$ dictates the exponential convergence rate, $\rho_{j, \infty}$ denotes the ultimate bound at the steady state and $\rho_{j, 0}$ is chosen to satisfy $\rho_{j o}>\left|e_{j}(0)\right|$. Hence, following the prescribed performance control methodology, ${ }^{19}$ the estimation law is designed as follows:

$$
\begin{aligned}
& \dot{\boldsymbol{p}}_{d}^{o} \triangleq\left[l_{1}, l_{2}, l_{3}\right]^{T} \\
& \boldsymbol{\omega}_{d}^{o} \triangleq 2 \boldsymbol{E}^{-1}\left(\eta_{d o}, \boldsymbol{\epsilon}_{d o}^{o}\right)\left[l_{4}, l_{5}, l_{6}\right]^{T}
\end{aligned}
$$

with

$$
l_{j} \triangleq-k_{j} \ln \left(\frac{1+\frac{e_{j}(t)}{\rho_{j}(t)}}{1-\frac{e_{j}(t)}{\rho_{j}(t)}}\right)+\frac{e_{j}(t)}{\rho_{j}(t)} \dot{\rho}_{j}(t), j=\{1, \ldots, 6\}
$$

and $k_{j}$ positive constants, from which the follower's estimates for position and orientation $\boldsymbol{p}_{d}, \boldsymbol{R}_{d}^{o}$ are calculated by integrating (26) and (27) using the expressions (2)-(3). Particularly, the integration of (27) will give the orientation in quaternions, which can be used to construct the rotation matrix $\boldsymbol{R}_{d}^{o}$. Moreover, we may calculate the desired acceleration signal by:

$$
\begin{gathered}
\ddot{\boldsymbol{p}}_{d}^{o} \triangleq\left[\dot{l}_{1}, \dot{l}_{2}, \dot{l}_{3}\right]^{T} \\
\dot{\boldsymbol{\omega}}_{d}^{o}=2 \dot{\boldsymbol{E}}^{-1}\left(\eta_{d o}, \boldsymbol{\epsilon}_{d o}^{o}\right)\left[l_{4}, l_{5}, l_{6}\right]^{T}+2 \boldsymbol{E}^{-1}\left(\eta_{d o}, \boldsymbol{\epsilon}_{d o}^{o}\right)\left[\dot{l}_{4}, \dot{l}_{5}, \dot{l}_{6}\right]^{T}
\end{gathered}
$$

where

$$
\dot{l}_{j}=\left(\dot{\rho}_{j}(t)-\frac{2 k_{j}}{1-\left(\frac{e_{j}(t)}{\rho_{j}(t)}\right)^{2}}\right) \frac{\dot{e}_{j}(t) \rho_{j}(t)-e_{j}(t) \dot{\rho}_{j}(t)}{\rho_{j}^{2}(t)}+\frac{e_{j}(t)}{\rho_{j}(t)} \ddot{\rho}_{j}(t)
$$

by simply differentiating (26), (27) with respect to time.

Theorem 1. Given a smooth and bounded desired trajectory $\boldsymbol{p}_{d_{l}}^{o}(t), \boldsymbol{R}_{d_{l}}^{o}(t)$ with bounded derivatives as well as the appropriately selected performance functions $\rho_{j}(t)$ for each element of $e(t)=\left[e_{1}(t), e_{2}(t), \ldots\right]^{T}$ that satisfy $\left|e_{j}(0)\right|<\rho_{j}(0)$ and incorporate the desired transient and steady-state performance specifications, the estimation laws (29) and (30) guarantee that $\left|e_{j}(t)\right|<\rho_{j}(t), \forall t \geq 0$.

Proof. Let us define the positive definite matrix $\boldsymbol{P}(t)=\operatorname{blockdiag}\left\{\boldsymbol{P}_{p}(t), \boldsymbol{P}_{r}(t)\right\}$, where $\boldsymbol{P}_{p}(t)=$ $\operatorname{diag}\left\{\frac{1}{\rho_{1}(t)}, \ldots \frac{1}{\rho_{3}(t)}\right\}, \boldsymbol{P}_{r}(t)=\operatorname{diag}\left\{\frac{1}{\rho_{4}(t)}, \ldots \frac{1}{\rho_{6}(t)}\right\}$; similarly we define the positive definite matrix $\boldsymbol{K}=\operatorname{blockdiag}\left\{\boldsymbol{K}_{p}, \boldsymbol{K}_{r}\right\}$ where $\boldsymbol{K}_{p}=\operatorname{diag}\left\{k_{1}, \ldots k_{3}\right\}, \boldsymbol{K}_{r}=\operatorname{diag}\left\{k_{4}, \ldots k_{6}\right\}$. Let us also define the normalized error:

$$
\boldsymbol{\xi}(t) \triangleq\left[\boldsymbol{\xi}_{p}(t)^{T}, \boldsymbol{\xi}_{\boldsymbol{r}}(t)^{T}\right]^{T}=\left[\xi_{1}(t), \ldots, \xi_{6}(t)\right]^{T}
$$

where

$$
\begin{aligned}
& \boldsymbol{\xi}_{p}(t)=\boldsymbol{P}_{p}(t) \boldsymbol{e}_{p}(t) \\
& \xi_{r}(t)=\boldsymbol{P}_{r}(t) \boldsymbol{e}_{r}(t) .
\end{aligned}
$$

Differentiating $\xi$ with respect to time and substituting (26)-(27) expressed in terms of the normalized error, we obtain $\dot{\boldsymbol{\xi}}=\boldsymbol{h}(t, \xi)=\left[\dot{\boldsymbol{\xi}}_{p}^{T}, \dot{\boldsymbol{\xi}}_{o}^{T}\right]^{T}$, with

$$
\begin{aligned}
& \dot{\boldsymbol{\xi}}_{p}(t) \triangleq-\boldsymbol{P}_{p}(t) \boldsymbol{K}_{p} \boldsymbol{\varepsilon}_{p}(t)-\boldsymbol{P}_{p}(t) \dot{\boldsymbol{p}}_{o}^{o}(t) \\
& \dot{\boldsymbol{\xi}}_{r}(t) \triangleq-\boldsymbol{P}_{r}(t) \boldsymbol{K}_{r} \boldsymbol{\varepsilon}_{r}(t)-\frac{1}{2} \boldsymbol{P}_{r}(t) \boldsymbol{E}\left(\eta_{d o}, \boldsymbol{\epsilon}_{d o}^{o}\right) \boldsymbol{\omega}_{o}^{o}(t)
\end{aligned}
$$

where $\boldsymbol{\varepsilon}_{p}(t)=\left[\varepsilon_{1}(t), \varepsilon_{2}(t), \varepsilon_{3}(t)\right]^{T}$ and $\boldsymbol{\varepsilon}_{o}(t)=\left[\varepsilon_{4}(t), \varepsilon_{5}(t), \varepsilon_{6}(t)\right]^{T}$ represent the transformed error signals for position and orientation, respectively, with

$$
\varepsilon_{j}(t)=\ln \left(\frac{1+\xi_{j}(t)}{1-\xi_{j}(t)}\right), j \in\{1, \ldots 6\}
$$


We also define the non-empty and open set

$$
\Omega_{\xi_{j}}=\underbrace{(-1,1) \times \cdots \times(-1,1)}_{6 \text { times }}
$$

In the sequel, we shall prove that $\xi(t)$ never escapes a compact subset of $\Omega_{\xi}$ and thus the performance bounds (24) are met. The following analysis is divided into two phases. First, we show that a maximal solution exists, such that $\xi(t) \in \Omega_{\xi} \forall t \in\left[0, \tau_{\max }\right)$, and subsequently we prove by contradiction that $\tau_{\max }$ is extended to $\infty$.

Phase A: Since $\left|e_{j}(0)\right|<\rho_{j}(0)$, we conclude that $\xi(0) \in \Omega_{\xi}$. Additionally, owing to the smoothness of: (a) the object's trajectory and (b) the proposed estimation scheme (26), (27) over $\Omega_{\xi}$, the function $\boldsymbol{h}(t, \xi)$ is continuous on $t \geq 0$ for all $\xi \in \Omega_{\xi}$. Therefore, the hypotheses of Theorem 54 (pp. 476 in ref. [20]) hold and the existence of a maximal solution $\xi(t)$ of (35), (36) on a time interval $\left[0, \tau_{\max }\right)$ such that $\xi(t) \in \Omega_{\xi}, \forall t \in\left[0, \tau_{\max }\right)$ is ensured.

Phase B: We have proven in Phase A that $\boldsymbol{\xi}(t) \in \Omega_{\xi}$. Therefore, the error signals $\boldsymbol{\varepsilon}_{p}(t), \boldsymbol{\varepsilon}_{o}(t)$ are well defined for all $t \in\left[0, \tau_{\max }\right)$. Hence, consider the positive definite and radially unbounded functions $V_{p}=\frac{1}{2} \boldsymbol{\varepsilon}_{p}^{T}(t) \boldsymbol{\varepsilon}_{p}(t), V_{r}=\frac{1}{2} \boldsymbol{\varepsilon}_{r}^{T}(t) \boldsymbol{\varepsilon}_{r}(t)$. Differentiating with respect to time and substituting (35) and (36), we obtain:

$$
\begin{aligned}
& \dot{V}_{p}(t)=\boldsymbol{\varepsilon}_{p}^{T}(t) \boldsymbol{\Xi}_{p}(t)\left(-\boldsymbol{K}_{p} \boldsymbol{P}_{p}(t) \boldsymbol{\varepsilon}_{p}(t)-\boldsymbol{P}_{p}(t) \dot{\boldsymbol{p}}_{o}^{o}(t)\right) \\
& \dot{V}_{r}(t)=\boldsymbol{\varepsilon}_{r}^{T}(t) \boldsymbol{\Xi}_{r}(t)\left(-\boldsymbol{K}_{r} \boldsymbol{P}_{r}(t) \boldsymbol{\varepsilon}_{r}(t)-\frac{1}{2} \boldsymbol{P}_{r} \boldsymbol{E}\left(\eta_{d o}, \boldsymbol{\epsilon}_{d o}^{o}\right) \boldsymbol{\omega}_{o}^{o}(t)\right)
\end{aligned}
$$

where $\boldsymbol{\Xi}_{p}=\operatorname{diag}\left\{\frac{1}{1-\xi_{j_{p}}^{2}}\right\}, j_{p}=\{1,2,3\}$ and $\boldsymbol{\Xi}_{r}=\operatorname{diag}\left\{\frac{1}{1-\xi_{j_{r}}^{2}}\right\}, j_{r}=\{4,5,6\}$. Owing to the fact that: (i) $\frac{1}{1-\xi_{j}^{2}}>1, \forall \xi_{j} \in \Omega_{\xi_{j}}$ and thus $\boldsymbol{\Xi}_{p}, \boldsymbol{\Xi}_{o}$ are positive definite, (ii) $\rho_{j}(t)>0, \forall t \geq 0$, (iii) $\boldsymbol{E}\left(\eta_{d o}, \boldsymbol{\epsilon}_{d o}^{o}\right)$ is bounded by construction and (iv) $\left|\dot{\boldsymbol{p}}_{o}^{o}(t)\right|,\left|\boldsymbol{\omega}_{o}^{o}(t)\right|$ are assumed bounded by an unknown positive constant $\bar{U}$, we conclude that $\dot{V}_{p}<0, \dot{V}_{r}<0$ when $\left|\varepsilon_{j}(t)\right|>\frac{\bar{U}}{\lambda_{\min }[K]}$ and consequently that:

$$
\left|\varepsilon_{j}(t)\right| \leq \bar{\varepsilon}_{j}=\max \left\{\left|\varepsilon_{j}(0)\right|, \frac{\bar{U}}{\lambda_{\min }[K]}\right\}, \forall t \in\left[0, \tau_{\max }\right) .
$$

Thus, invoking the inverse of (37), we get:

$$
-1<\frac{e^{-\bar{\varepsilon}_{j}}-1}{e^{-\bar{\varepsilon}_{j}}+1}=\underline{\xi}_{j} \leq \xi_{j}(t) \leq \bar{\xi}_{j}=\frac{e^{\bar{\varepsilon}_{j}}-1}{e^{\bar{\varepsilon}_{j}}+1}<1 .
$$

Therefore, $\xi_{j}(t) \in \Omega_{\xi_{j}}^{\prime}=\left[\underline{\xi}_{j}, \bar{\xi}_{j}\right], \forall t \in\left[0, \tau_{\max }\right)$, which is a nonempty and compact subset of $\Omega_{\xi_{j}}$. Hence, assuming $\tau_{\max }<\infty$ and since $\Omega_{\xi_{j}}^{\prime} \subset \Omega_{\xi_{j}}$, Proposition C.3.6 (pp. 481 in ref. [20]) dictates the existence of a time instance $t^{\prime} \in\left[0, \tau_{\max }\right)$ such that $\xi_{j}\left(t^{\prime}\right) \notin \Omega_{\xi_{j}}^{\prime}$, which is a clear contradiction. Therefore, $\tau_{\max }$ is extended to $\infty$. As a result, all closed loop signals remain bounded and moreover $\xi_{j}(t) \in \Omega_{\xi_{j}}^{\prime} \subset \Omega_{\xi_{j}}, \forall t \geq 0$. Finally, from (33), (34) and (41), we conclude that:

$$
-\rho_{j}(t)<\underline{\xi}_{j} \rho_{j}(t) \leq e_{j}(t) \leq \bar{\xi}_{j} \rho_{j}(t)<\rho_{j}(t)
$$

for all $t \geq 0$, which completes the proof.

Remark 1. The proposed estimation scheme is more robust against desired trajectory profiles with non-zero acceleration than previous works presented in refs. [21-23]. The only necessary condition concerns the smoothness and boundedness of the desired trajectory. In this sense, our method guarantees bounded closed-loop signals and practical asymptotic stabilization of the estimation errors. 
3.2. Single manipulator impedance control

An impedance control scheme will be employed based on the desired trajectory estimation presented in Section 3.1. The desired impedance model is selected as:

$$
\begin{gathered}
\boldsymbol{M}_{d_{p}} \Delta \ddot{\boldsymbol{p}}_{d o}^{o}+\boldsymbol{K}_{d_{p}} \Delta \dot{\boldsymbol{p}}_{d o}^{o}+\boldsymbol{K}_{p_{p}} \Delta \boldsymbol{p}_{d o}^{o}=-\boldsymbol{f}_{l o}^{o} \\
\boldsymbol{M}_{d_{r}} \Delta \dot{\boldsymbol{\omega}}_{d o}^{o}+\boldsymbol{K}_{d_{r}} \Delta \boldsymbol{\omega}_{d o}^{o}+\boldsymbol{K}_{p_{r}}^{\prime} \boldsymbol{\epsilon}_{d o}^{o}=-\boldsymbol{\mu}_{l o}^{o}
\end{gathered}
$$

with

$$
\boldsymbol{K}_{p_{r}}^{\prime}=2 \boldsymbol{E}\left(\eta_{d o}, \boldsymbol{\epsilon}_{d o}^{o}\right)^{T} \boldsymbol{K}_{p_{r}}
$$

where $\boldsymbol{M}_{d_{p}}, \boldsymbol{M}_{d_{r}}, \boldsymbol{K}_{d_{p}}, \boldsymbol{K}_{d_{r}}, \boldsymbol{K}_{p_{p}}$ and $\boldsymbol{K}_{p_{r}}^{\prime}$ are appropriate gain matrices corresponding to inertia, damping and stiffness according to ref. [24]. Although it is omitted for simplicity, it is advisable to follow the geometrical consistent active stiffness contribution ${ }^{25}$ for the choice of the stiffness matrix of the desired impedance.

Since the measurement of $\boldsymbol{h}_{l}^{o}$ is not available, an estimation $\hat{\boldsymbol{h}}_{l}^{o}$ should be provided. Hence, we exploit: (a) the ultimate boundedness of the human motion intention estimation error $\boldsymbol{e}(t)$, (b) the inertial and geometric parameters of the object, which are assumed known, as well as (c) the dynamic equation of the object (17), to estimate it by:

$$
-\hat{\boldsymbol{h}}_{l o}^{o}=-\boldsymbol{J}_{o l}^{T} \hat{\boldsymbol{h}}_{l}^{o}=\boldsymbol{M}_{o} \dot{\boldsymbol{v}}_{d}^{o}+\boldsymbol{C}_{o}\left(\boldsymbol{v}_{o}^{o}\right) \boldsymbol{v}_{o}^{o}+\boldsymbol{G}_{o}+\boldsymbol{G} \boldsymbol{H}_{f}^{o}
$$

Equivalently, invoking (17), we obtain:

$$
\begin{aligned}
-\boldsymbol{J}_{o l}^{T} \boldsymbol{h}_{l}^{o} & =\boldsymbol{M}_{o} \dot{\boldsymbol{v}}_{o}^{o}+\boldsymbol{C}_{o}\left(\boldsymbol{v}_{o}^{o}\right) \boldsymbol{v}_{o}^{o}+\boldsymbol{G}_{o}+\boldsymbol{G} \boldsymbol{H}_{f}^{o} \\
& =\boldsymbol{M}_{o} \dot{\boldsymbol{v}}_{d}^{o}+\boldsymbol{C}_{o}\left(\boldsymbol{v}_{o}^{o}\right) \dot{\boldsymbol{v}}_{o}^{o}+\boldsymbol{G}_{o}+\boldsymbol{G} \boldsymbol{H}_{f}^{o}+\boldsymbol{M}_{o} \Delta \dot{\boldsymbol{v}}_{d o}^{o} \\
& =-\boldsymbol{J}_{o l}^{T} \hat{\boldsymbol{h}}_{l}^{o}+\boldsymbol{M}_{o} \Delta \dot{\boldsymbol{v}}_{d o}^{o} \\
-\boldsymbol{h}_{l o}^{o} & =-\hat{\boldsymbol{h}}_{l o}^{o}+\boldsymbol{M}_{o} \Delta \dot{\boldsymbol{v}}_{d o}^{o}
\end{aligned}
$$

Thus, selecting $\boldsymbol{M}_{d_{p}}$ and $\boldsymbol{M}_{d_{r}}$ such that blockdiag $\left\{\boldsymbol{M}_{d_{p}}^{*}, \boldsymbol{M}_{d_{r}}^{*}\right\}=\operatorname{blockdiag}\left\{\boldsymbol{M}_{d_{p}}, \boldsymbol{M}_{d_{r}}\right\}-$ blockdiag $\left\{\boldsymbol{M}_{o_{p}}, \boldsymbol{M}_{o_{r}}\right\}$ is positive definite, the desired impedance can be expressed as

$$
\begin{aligned}
& \boldsymbol{M}_{d_{p}}^{*} \Delta \ddot{\boldsymbol{p}}_{d o}^{o}+\boldsymbol{K}_{d_{p}} \Delta \dot{\boldsymbol{p}}_{d o}^{o}+\boldsymbol{K}_{p_{p}} \Delta \boldsymbol{p}_{d o}^{o}=-\hat{\boldsymbol{f}}_{l o}^{o} \\
& \boldsymbol{M}_{d_{r}}^{*} \Delta \dot{\boldsymbol{\omega}}_{d o}^{o}+\boldsymbol{K}_{d_{r}} \Delta \boldsymbol{\omega}_{d o}^{o}+\boldsymbol{K}_{p_{r}} \boldsymbol{\epsilon}_{d o}^{o}=-\hat{\boldsymbol{\mu}}_{l o}^{o}
\end{aligned}
$$

Let us also express for later convenience the Eqs. (47) and (48) in compact matrix form:

$$
\boldsymbol{M}_{d}^{*} \Delta \ddot{\boldsymbol{v}}_{d o}^{o}+\boldsymbol{K}_{d} \Delta \dot{\boldsymbol{v}}_{d o}^{o}+\boldsymbol{K}_{p} \Delta \boldsymbol{x}_{d_{l o}}^{o}=-\hat{\boldsymbol{h}}_{l o}^{o}
$$

Notice that in case of a single manipulator $\boldsymbol{G}=\boldsymbol{J}_{o f}^{T}$, thus the grasp matrix is full rank. Notice also that in case of unknown or varying leader's grasping point, $\boldsymbol{J}_{o l}$ can be interpreted as a $(6 \times 6)$ identity matrix. This can be justified by the fact that the provided $\mathrm{f} / \mathrm{t}$ measurement can be transformed to $\Sigma_{o}$ and thus the actual grasping point does not matter as long as large internal forces and singular configurations due to rigid grasping constraint are avoided by the human during the interaction. Finally, we design the inverse dynamics control law:

$$
\boldsymbol{u}_{f}=\boldsymbol{J}_{o f}^{-T}\left(\boldsymbol{M}_{o f}\left(\boldsymbol{q}_{f}\right) \boldsymbol{\alpha}+\boldsymbol{C}_{o f}\left(\dot{\boldsymbol{q}}_{f}, \boldsymbol{q}_{f}\right) \boldsymbol{v}_{o}+\boldsymbol{G}_{o f}\left(\boldsymbol{q}_{f}\right)-\boldsymbol{J}_{o f}^{T} \boldsymbol{h}_{f}\right)
$$

to enforce the desired impedance behavior, where $\boldsymbol{\alpha}=\left[\boldsymbol{\alpha}_{p}^{T}, \boldsymbol{\alpha}_{r}^{T}\right]$ refers to the resolved acceleration. By differentiating $\boldsymbol{p}_{o}=\boldsymbol{R}_{o} \boldsymbol{p}_{o}^{o}$ twice and $\boldsymbol{\omega}_{o}=\boldsymbol{R}_{o} \boldsymbol{\omega}_{o}^{o}$ we get

$$
\dot{\boldsymbol{v}}_{o}=\left[\begin{array}{c}
\boldsymbol{R}_{o} \ddot{\boldsymbol{p}}_{o}^{o}+2 \dot{\boldsymbol{S}}\left(\boldsymbol{\omega}_{o}\right) \boldsymbol{R}_{o} \dot{\boldsymbol{p}}_{o}^{o}+\boldsymbol{S}\left(\dot{\boldsymbol{\omega}}_{o}\right) \boldsymbol{p}_{o}+\boldsymbol{S}\left(\boldsymbol{\omega}_{o}\right) \boldsymbol{S}\left(\boldsymbol{\omega}_{o}\right) \boldsymbol{p}_{o} \\
\boldsymbol{R}_{o} \dot{\boldsymbol{\omega}}_{o}^{o}+\boldsymbol{S}\left(\boldsymbol{\omega}_{o}\right) \boldsymbol{\omega}_{o}
\end{array}\right]
$$


Hence, choosing

$$
\boldsymbol{\alpha}=\left[\begin{array}{c}
\boldsymbol{\alpha}_{p} \\
\boldsymbol{\alpha}_{r}
\end{array}\right]=\left[\begin{array}{c}
\boldsymbol{R}_{o} \boldsymbol{\alpha}_{p}^{o}+2 \dot{\boldsymbol{S}}\left(\omega_{o}\right) \boldsymbol{R}_{o} \dot{\boldsymbol{p}}_{o}^{o}+\boldsymbol{S}\left(\dot{\boldsymbol{\omega}}_{o}\right) \boldsymbol{p}_{o}+\boldsymbol{S}\left(\boldsymbol{\omega}_{o}\right) \boldsymbol{S}\left(\boldsymbol{\omega}_{o}\right) \boldsymbol{p}_{o} \\
\boldsymbol{R}_{o} \boldsymbol{\alpha}_{r}^{o}+\boldsymbol{S}\left(\boldsymbol{\omega}_{o}\right) \boldsymbol{\omega}_{o}
\end{array}\right]
$$

with

$$
\begin{aligned}
\boldsymbol{\alpha}_{p}^{o} & =\ddot{\boldsymbol{p}}_{d}^{o}+\boldsymbol{M}_{d_{p}}^{*-1}\left(\boldsymbol{K}_{d_{p}} \Delta \dot{\boldsymbol{p}}_{d o}^{o}+\boldsymbol{K}_{p_{p}} \Delta \boldsymbol{p}_{d o}^{o}+\hat{\boldsymbol{f}}_{l o}^{o}\right) \\
\boldsymbol{\alpha}_{r}^{o} & =\dot{\boldsymbol{\omega}}_{d}^{o}+\boldsymbol{M}_{d_{r}}^{*-1}\left(\boldsymbol{K}_{d_{r}} \Delta \boldsymbol{\omega}_{d o}^{o}+\boldsymbol{K}_{p_{r}}^{\prime} \boldsymbol{\epsilon}_{d o}^{o}+\hat{\boldsymbol{\mu}}_{l o}^{o}\right)
\end{aligned}
$$

we achieve the desired impedance. To proceed with the stability analysis of the aforementioned resolved acceleration laws, we define first the energy-based Lyapunov function:

$$
V=\frac{1}{2} \Delta \dot{\boldsymbol{p}}_{d o}^{o T} \boldsymbol{M}_{d_{p}}^{*} \Delta \dot{\boldsymbol{p}}_{d o}^{o}+\frac{1}{2} \Delta \dot{\boldsymbol{\omega}}_{d o}^{o T} \boldsymbol{M}_{d_{r}}^{*} \Delta \boldsymbol{\omega}_{d o}^{o}+\frac{1}{2} \boldsymbol{p}_{d o}^{o T} \boldsymbol{K}_{p_{p}} \boldsymbol{p}_{d o}^{o}+2 \boldsymbol{\epsilon}_{d o}^{o T} \boldsymbol{K}_{p_{r}} \boldsymbol{\epsilon}_{d o}^{o}
$$

Differentiating with respect to time and substituting the control law (50), we get:

$$
\dot{V}=-\Delta \dot{\boldsymbol{p}}_{d o}^{o T} \boldsymbol{K}_{d_{p}} \Delta \dot{\boldsymbol{p}}_{d o}^{o}-\Delta \boldsymbol{\omega}_{d o}^{o T} \boldsymbol{K}_{d_{r}} \Delta \boldsymbol{\omega}_{d o}^{o}-\Delta \dot{\boldsymbol{p}}_{d o} \hat{\boldsymbol{f}}_{l o}^{o}-\Delta \boldsymbol{\omega}_{d o}^{o T} \hat{\boldsymbol{\mu}}_{l o}^{o}
$$

which in the absence of external wrenches is negative semidefinite. Hence, we conclude (see ref. [26] for detailed proof) that in the absence of human wrenches, the system is asymptotically stable and tracking of the desired trajectory is achieved. In the presence of human wrenches, the term $-\Delta \dot{\boldsymbol{p}}_{d o}^{o} \hat{\boldsymbol{f}}_{l o}^{o}-\Delta \boldsymbol{\omega}_{d o}^{o T} \hat{\boldsymbol{\mu}}_{l o}^{o}$ denotes the power exerted by the human on the system, which shapes the energy of the system and defines new stable trajectories. Hence, the lower the human wrench exerted on the object, the smaller the deviations from the desired trajectories will be.

\subsection{Unknown object dynamics}

Object identification is, in general, a difficult and time-consuming task; thus, only a rough estimate of the dynamical model of the manipulated object is available. In this subsection, we design an adaptive scheme to deal with the unavoidable uncertainties and thus increase the robustness of the closed-loop system. Hence, assuming unknown object dynamics, the impedance reference model (47) and (48) can be written as:

$$
\begin{array}{r}
\boldsymbol{M}_{d_{p}}^{*} \Delta \ddot{\boldsymbol{p}}_{d o}^{o}+\boldsymbol{K}_{d_{p}} \Delta \dot{\boldsymbol{p}}_{d o}^{o}+\boldsymbol{K}_{p_{p}} \Delta \boldsymbol{p}_{d o}^{o}=-\tilde{\boldsymbol{f}}_{l o}^{o} \\
\boldsymbol{M}_{d_{r}}^{*} \Delta \dot{\boldsymbol{\omega}}_{d}^{o}+\boldsymbol{K}_{d_{r}} \Delta \boldsymbol{\omega}_{d}^{o}+\boldsymbol{K}_{p_{r}} \boldsymbol{\epsilon}_{d o}^{o}=-\tilde{\boldsymbol{\mu}}_{l o}^{o}
\end{array}
$$

where $\tilde{\boldsymbol{f}}_{l}^{o}, \tilde{\boldsymbol{\mu}}_{l}^{o}$ denotes the estimated human wrench due to the uncertainty in both the desired trajectory and the object's parameters. Invoking (23) and using the estimated acceleration $\dot{\boldsymbol{v}}_{d}^{o}$, we obtain:

$$
\boldsymbol{Y}\left(\boldsymbol{v}_{o}^{o}, \dot{\boldsymbol{v}}_{d}^{o}\right) \boldsymbol{\theta}=-\boldsymbol{h}_{f o}^{o}-\hat{\boldsymbol{h}}_{l o}^{o}
$$

In the presence of parametric uncertainty, the above equation is modified to

$$
\boldsymbol{Y}\left(\boldsymbol{v}_{o}^{o}, \dot{\boldsymbol{v}}_{d}^{o}\right) \hat{\boldsymbol{\theta}}=-\boldsymbol{h}_{f o}^{o}-\tilde{\boldsymbol{h}}_{l o}^{o}
$$

Thus, the impedance reference model becomes

$$
\begin{array}{r}
\boldsymbol{M}_{d_{p}}^{*} \Delta \ddot{\boldsymbol{p}}_{d o}^{o}+\boldsymbol{K}_{d_{p}} \Delta \dot{\boldsymbol{p}}_{d o}^{o}+\boldsymbol{K}_{p_{p}} \Delta \boldsymbol{p}_{d o}^{o}=\boldsymbol{Y}_{p}\left(\boldsymbol{v}_{o}^{o}, \dot{\boldsymbol{v}}_{d}^{o}\right) \hat{\boldsymbol{\theta}}+\boldsymbol{f}_{f o}^{o} \\
\boldsymbol{M}_{d_{r}}^{*} \Delta \dot{\boldsymbol{\omega}}_{d o}^{o}+\boldsymbol{K}_{d_{r}} \Delta \boldsymbol{\omega}_{d o}^{o}+\boldsymbol{K}_{p_{r}} \boldsymbol{\epsilon}_{d o}^{o}=\boldsymbol{Y}_{r}\left(\boldsymbol{v}_{o}^{o}, \dot{\boldsymbol{v}}_{d}^{o}\right) \hat{\boldsymbol{\theta}}+\boldsymbol{\mu}_{f o}^{o}
\end{array}
$$

Following the same control strategy presented in (50)-(54) with the parameters of the object updated by

$$
\dot{\hat{\boldsymbol{\theta}}}=-\boldsymbol{\Gamma}^{-T}\left(\boldsymbol{Y}_{p}^{T} \Delta \dot{\boldsymbol{p}}_{d o}^{o}+\boldsymbol{Y}_{o}^{T} \Delta \boldsymbol{\omega}_{d o}^{o}\right), \boldsymbol{\Gamma}>0 .
$$


and by adopting the Lyapunov function candidate:

$$
V=\frac{1}{2} \Delta \dot{\boldsymbol{p}}_{d o}^{o T} \boldsymbol{M}_{d_{p}}^{*} \Delta \dot{\boldsymbol{p}}_{d o}^{o}+\frac{1}{2} \Delta \dot{\boldsymbol{\omega}}_{d o}^{o T} \boldsymbol{M}_{d_{r}}^{*} \Delta \boldsymbol{\omega}_{d o}^{o}+\frac{1}{2} \boldsymbol{p}_{d o}^{o T} \boldsymbol{K}_{p_{p}} \boldsymbol{p}_{d o}^{o}+2 \boldsymbol{\epsilon}_{d o}^{o T} \boldsymbol{K}_{p_{r}} \boldsymbol{\epsilon}_{d o}^{o}+\frac{1}{2} \tilde{\boldsymbol{\theta}}^{T} \boldsymbol{\Gamma} \tilde{\boldsymbol{\theta}}
$$

with $\tilde{\boldsymbol{\theta}}=\boldsymbol{\theta}-\hat{\boldsymbol{\theta}}$, we arrive at (56). Thus, the same stability analysis holds.

\subsection{Multiple manipulators impedance control}

The control methodology for a single manipulator can be extended for multiple robots at the cost of inter-robot communication for the measurements of each $\mathrm{f} / \mathrm{t}$ sensor ${ }^{3}$. Moreover, as the system is dynamically constrained by the rigid grasp property, any inconsistencies between the manipulators' trajectories may yield internal forces which should be avoided. Thus, based on ref. [28], a stabilization scheme was adopted. Substituting Eqs. (19) and (22) into (15), we obtain

$$
\boldsymbol{M}_{O F} \dot{\boldsymbol{V}}_{o}+\boldsymbol{C}_{O F} \boldsymbol{V}_{o}+\boldsymbol{G}_{O F}=\boldsymbol{J}_{O F}^{T}(\boldsymbol{U})+\boldsymbol{J}_{O F}^{T}\left(\overline{\boldsymbol{G}} \boldsymbol{G} \boldsymbol{H}_{f}^{o}+\boldsymbol{V} \boldsymbol{h}_{I}\right)
$$

Under the assumption of full row rank grasp matrix, it is shown that the following control law

$$
\boldsymbol{U}=\boldsymbol{J}_{O F}^{-T}\left(\boldsymbol{M}_{O F} \boldsymbol{J}_{S}^{T} \boldsymbol{\alpha}+\boldsymbol{C}_{O F} \boldsymbol{V}_{o}+\boldsymbol{G}_{O F}-\boldsymbol{J}_{o f}^{T} \overline{\boldsymbol{G}} \boldsymbol{G} \boldsymbol{H}_{f}^{o}\right)-\boldsymbol{J}_{O F}^{T} \boldsymbol{V}\left(\boldsymbol{h}_{I d}+\boldsymbol{K}_{f} \int\left(\boldsymbol{h}_{I d}-\boldsymbol{h}_{I}\right) d t\right)
$$

with $\boldsymbol{J}_{S}=\left[\begin{array}{l}\boldsymbol{I} \\ \boldsymbol{I}\end{array} \cdots \boldsymbol{I}\right]$ and $\boldsymbol{\alpha}$ as defined in (52)-(54) establishes the desired impedance and guarantees that $\boldsymbol{h}_{I}$ converges to the desired internal force $\boldsymbol{h}_{I d}$. In particular, substituting Eq. (66) into (15) we obtain:

$$
\begin{aligned}
\boldsymbol{M}_{O F} \boldsymbol{J}_{S}^{T}\left(\dot{\boldsymbol{v}}_{d o}^{o}+\boldsymbol{M}_{d}^{*-1}\left(\boldsymbol{K}_{d} \Delta \dot{\boldsymbol{v}}_{d o}^{o}\right.\right. & \left.\left.+\boldsymbol{K}_{p} \Delta \boldsymbol{x}_{d o}^{o}-\hat{\boldsymbol{h}}_{l}^{o}\right)\right) \\
& +\boldsymbol{J}_{O F}^{T} \boldsymbol{V}\left(\left(\boldsymbol{h}_{I}-\boldsymbol{h}_{I d}\right)+\boldsymbol{K}_{f} \int\left(\boldsymbol{h}_{I}-\boldsymbol{h}_{I d}\right) d t\right)=\mathbf{0} .
\end{aligned}
$$

Pre-multiplying (67) by $\boldsymbol{G} \boldsymbol{J}_{O F}^{-T}$ and invoking the fact that the range space of $\boldsymbol{V}$ is the null space of $\boldsymbol{G}$ (i.e., $\boldsymbol{G} \boldsymbol{V}=0$ ), we obtain:

$$
\boldsymbol{G} \boldsymbol{J}_{O F}^{-T} \boldsymbol{M}_{O F} \boldsymbol{J}_{S}^{T}\left(\Delta \dot{\boldsymbol{v}}_{d o}^{o}+\boldsymbol{M}_{d}^{*-1}\left(\boldsymbol{K}_{d} \Delta \boldsymbol{v}_{d o}^{o}+\boldsymbol{K}_{p} \Delta \boldsymbol{x}_{d o}^{o}-\hat{\boldsymbol{h}}_{l}^{o}\right)\right)=\mathbf{0} .
$$

Moreover, $\boldsymbol{G} \boldsymbol{J}_{O F}^{-T} \boldsymbol{M}_{O F} \boldsymbol{J}_{S}^{T}$ is non-singular. Hence, the resulting system is given by:

$$
\boldsymbol{M}_{d}^{*} \Delta \ddot{\boldsymbol{v}}_{d o}^{o}+\boldsymbol{K}_{d} \Delta \boldsymbol{v}_{d o}^{o}+\boldsymbol{K}_{p} \Delta \boldsymbol{x}_{d o}^{o}=\hat{\boldsymbol{h}}_{l o}^{o}
$$

which is the stable system with the desired impedance, for which the analysis of Section 3.2 holds. Substituting Eq. (67) into (49) under the assumption that $\boldsymbol{J}_{O F}$ is non-singular and that $V$ is always full rank, we obtain:

$$
\left(\boldsymbol{h}_{I}-\boldsymbol{h}_{I d}\right)+\boldsymbol{K}_{f} \int\left(\boldsymbol{h}_{I}-\boldsymbol{h}_{I d}\right) d t=\mathbf{0}
$$

which implies that $\boldsymbol{h}_{I}$ converges to $\boldsymbol{h}_{I d}$.

\section{Experimental Results}

The experimental evaluation of the proposed method was performed using a Kinova Mico2 6DoF robotic manipulator. An interface was developed to allow high-frequency communication, using Kinova's low-level API. The interface was then wrapped using the ros-control package. The proposed control law was implemented as a new ros-controller, running on a Linux system at $220 \mathrm{~Hz}$. The manipulator's sensor suite provides measurements of position, velocity and torque at each joint. From the torque measurements, we estimated the wrenches acting at the end effector by employing the robot model and the acceleration estimation from our controller. The calculated wrench was

${ }^{3} \mathrm{~A}$ force consensus strategy as in ref. [27] could be employed to relax the inter-robot communication requirement. A purely decentralized method for the N-robots scenario is left open for future work. 
(a)

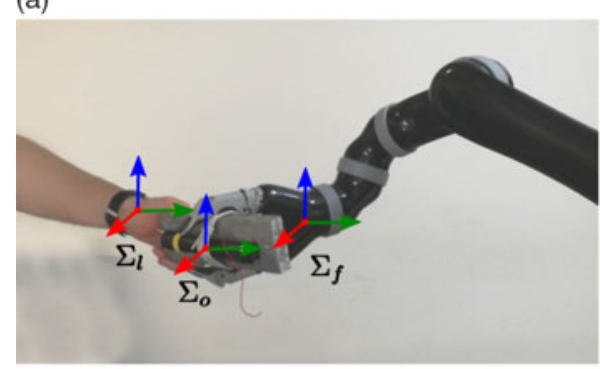

(b)

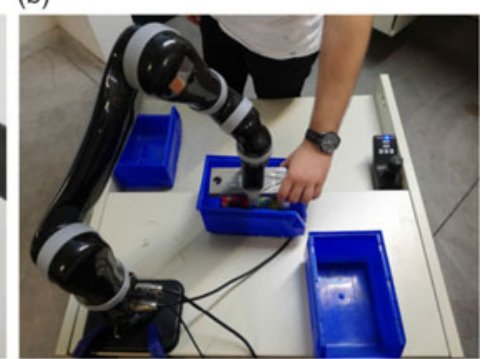

(c)

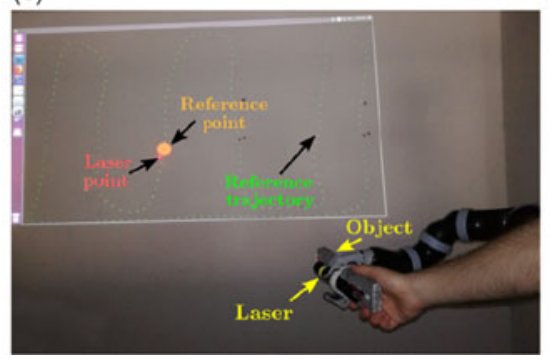

Fig. 3. (a) Cooperative transportation setup, (b) example of a task as described in the experiment of Section 4.1, the subject had to place the object inside every blue box starting from an arbitrary position, (c) the setup used for the experiments described in Section 4.2.

remarkably close to the wrench estimation obtained by Kinova's built-in software, which is not available when operating with the low-level API. Both APIs provide functionality that allows to directly send torque commands $(\mathrm{Nm})$ to the motors. Therefore, the control commands are directly passed to the motors. Finally, it should be noted that the purpose of the experimental evaluation of this work is twofold. Hence, two different experiments have been designed and implemented. The first is related to the verification of the cooperative transportation concept, while the second attempts to quantify the human effort reduction.

\subsection{Cooperative transportation verification}

This experiment examines if the proposed method results in a smooth, intuitive interaction with the human leader and does not compromise the manipulation ability that is, the human leader should be able to implement the same tasks he can accomplish by operating the manipulator in gravity compensation mode using the same level of mental effort. Toward this direction, a set of experiments that were carried out by 10 subjects, all adults between 24 and 35 years old was designed. The experimental setup is presented in Fig. 3(a) and (b), where an object grasped by the manipulator had to be transported collaboratively by the robot and a human leader to perform 6D-trajectories via certain checkpoints in the form of a task. Such tasks may involve simple assignments commonly met in collaborative transportation such as peg-in-hole, pick-and-place and obstacle avoidance. The same tasks were also performed holding the robot arm (and the object) in gravity compensation mode just for comparison purposes. Apparently, our setup does not allow quantifiable results for $6 \mathrm{DoF}$ trajectory tracking as the ones presented in ref. [29] ${ }^{4}$ for $1 \mathrm{DoF}$ due to the lack of means to create a $6 \mathrm{DoF}$ reference trajectory (i.e., augmented reality). In that sense, the results of this experimental study are qualitative. Before starting the experiments, the subjects needed some time to familiarize themselves with the setup, that is, implement random trajectories. The time required for this procedure spanned from seconds to a few minutes. After this step, each subject was able to complete the given tasks without any obvious difficulty. Finally, the subjects were asked to rate the experience of the interaction in terms of convenience, cooperation and mental effort, as compared to the interaction during the execution of the same tasks in gravity compensation mode. In gravity compensation operation,

${ }^{4}$ Regarding ref. [29], although the proposed estimator remains the same, the impedance control formulation is different for consistency with the extended experimental verification that was carried out in this work. Moreover, an adaptive law that compensates for uncertainties in object modeling and a multi-robot scenario have been further considered in this work. 
Table I. Cooperative transportation qualitative outcome.

\begin{tabular}{lccc}
\hline & $\begin{array}{c}\text { Familiarization } \\
\text { time (min) }\end{array}$ & $\begin{array}{c}\text { Successful } \\
\text { attempts }\end{array}$ & $\begin{array}{c}\text { Interaction } \\
\text { rating }\end{array}$ \\
\hline Subject 1 & 1.5 & $5 / 5$ & $9 / 10$ \\
Subject 2 & 3.0 & $5 / 5$ & $10 / 10$ \\
Subject 3 & 4.5 & $5 / 5$ & $8 / 10$ \\
Subject 4 & 2.0 & $5 / 5$ & $9 / 10$ \\
Subject 5 & 2.0 & $5 / 5$ & $7 / 10$ \\
Subject 6 & 1.0 & $5 / 5$ & $9 / 10$ \\
Subject 7 & 1.5 & $5 / 5$ & $7 / 10$ \\
Subject 8 & 1.0 & $5 / 5$ & $8 / 10$ \\
Subject 9 & 3.0 & $4 / 5$ & $8 / 10$ \\
Subject 10 & 1.5 & $5 / 5$ & $9 / 10$ \\
\hline
\end{tabular}

the subjects had no difficulty in completing any given task and no familiarization time was required. The results are presented in Table I, while relevant demonstrations can be found at https://vimeo. com/283025257 alongside the rest of the experimental study.

Notice that as long as our setup is equipped with torque measurements in each joint, the human leader can interact with any part of the robot or the object with the same results. Moreover, $\boldsymbol{J}_{o l}$ is not utilized in the control law since $\boldsymbol{h}_{l o}^{o}$ can be directly computed by ref. [45]. Notice that it is convenient to compute all wrenches to the object's center of mass to avoid the uncertainty in the case of varying contact points, by-passing completely $\boldsymbol{J}_{o l}$. Therefore, the human can use multiple grasping points or deliberately lose contact with the robot (or the object) and reconfigure his/her arm to avoid joint limits (as long as the contact points allow f/t measurement).

The results of Table I reveal that no significant training is required for the human leader to complete a variety of common tasks (mean value: $126 \mathrm{~s}$, standard deviation: $66 \mathrm{~s}$ ). Hence, the subjects were able to impose their desired trajectory, which indicates a successful application of the estimation law. Moreover, the rating on the interaction experience is high (mean value: 8.4/10, standard deviation: 0.96) which means that the quality of interaction perceived from the convenience and intuitive behavior points of view is comparable to gravity compensation. However, to our view, the explanation to this mainly lies in reasons related to noisy torque measurements, imperfect modeling of the manipulator (which in turn hinders the end effector wrench estimation) and the unusual experience of interacting with an underdamped articulated body, rather than on the estimation procedure. To elaborate on the above statement, we mention that when we imposed a more conservative tuning to the impedance controller (less leader wrench augmentation, less damping), there was -as expectedno effect on the estimation, although the interaction was obviously more convenient and very close to gravity compensation. On the other hand, the effort reduction was suppressed and, as a result, the tuning was a trade-off between effort reduction and the desired interaction.

Figure 4 presents the performance of the estimation law. Human intention estimation is considered the key ingredient of the proposed approach and its integration with the impedance controller constitutes the main contribution of this work. As shown, the estimation of the desired human motion tracks the actual motion of the robot, without violating the performance bounds, which can be interpreted as a successful application of the estimation law. Velocity estimation is also close to the actual one and significantly smoother. Notice that the actual acceleration signal in Fig. 4 is not used in the proposed methodology, and it is presented here for comparison. Moreover, as there is no acceleration measurement available, the actual velocity signal was numerically differentiated and then smoothed, using zero-phase filtering, for illustration purposes. From the acceleration signals in Fig. 4, it is derived that the estimation is adequately close to the "actual" acceleration and although it is not depicted, it is obvious that the estimated acceleration is substantially smoother than a simple differentiation of the actual velocity would have been. From ref. (46), it follows that the accuracy of estimating human wrench $\hat{\boldsymbol{h}}_{l o}$ actually depends on the inertial model's accuracy of the object and the accuracy of the 

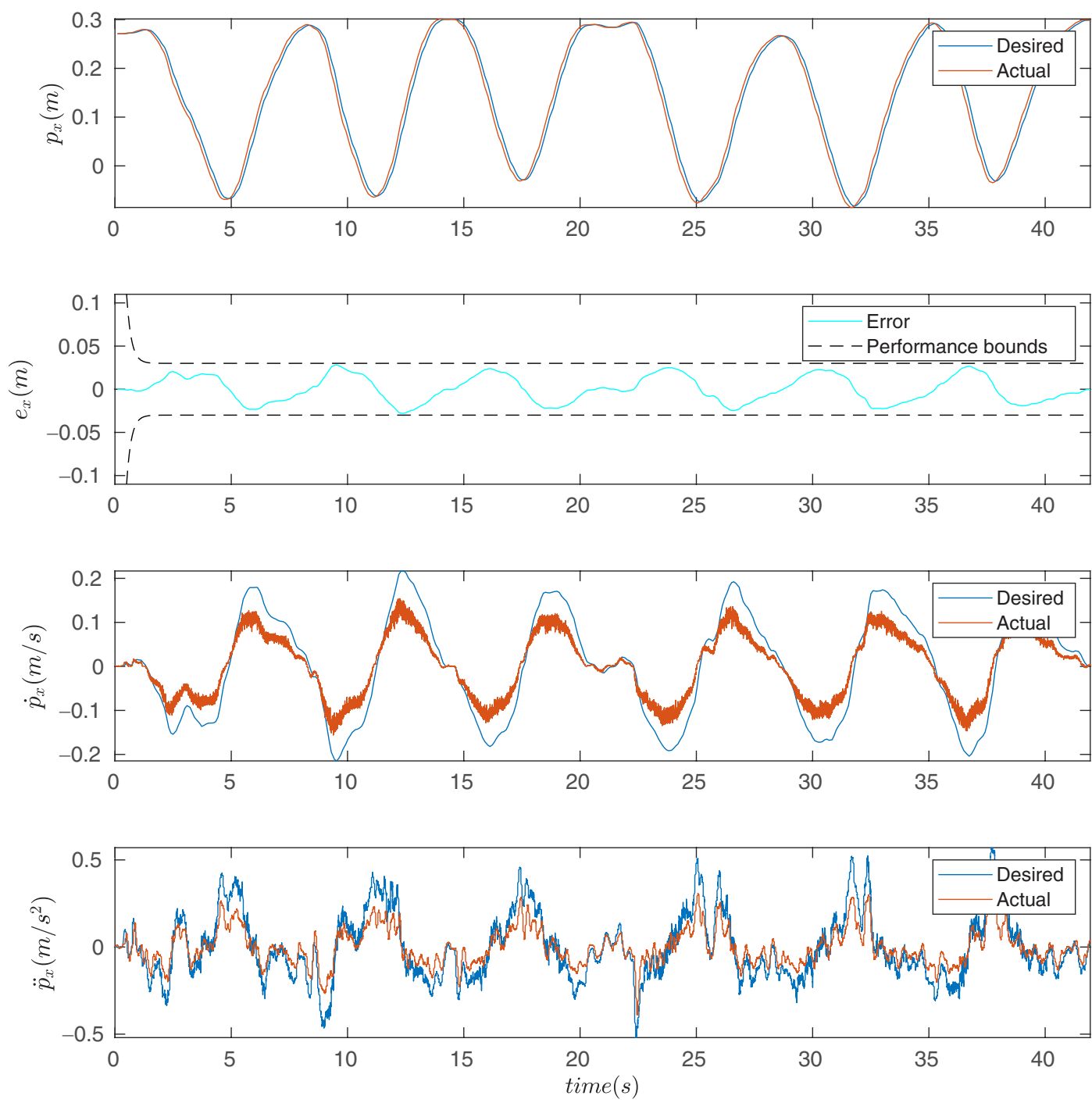

Fig. 4. Example of estimation in position, velocity and acceleration where desired is referring to the estimated signal and actual to the measured one.

acceleration estimation which is depicted in Fig. 4. As shown in Fig. 5 concerning similar trajectories, the force attributed to the object's motion does not differ noticeably in both methods. However, the interaction force is significantly reduced.

\subsection{Human effort reduction}

After the successful assessment of the first results, the second set of experiments aims to quantitatively elaborate on the concept of human effort reduction during a collaborative transportation task. To this end, the setup depicted in Fig. 3(c) was introduced. Multiple 2D trajectories of a reference point at different speed profiles were generated and projected on a vertical surface. The carried object had a laser attached to it, the dynamic parameters of both the object and the laser, as a single object, were identified and are presented in Table II. It is worth noting that the same object was used in the first experiments. The human leader should collaboratively transport an object with the manipulator such that the laser beam tracks the projected reference point. The subjects ran the same experiments for both the proposed approach and gravity compensation by performing a set of 7 to 10 trajectories for each method. The parameters of the proposed scheme in both experiments were chosen as shown in Table III. $\boldsymbol{M}_{d}^{*}, \boldsymbol{K}_{d}$, and $\boldsymbol{K}_{p}$ are components of a simple impedance controller and thus are tuned accordingly. Specifically, $\boldsymbol{M}_{d}^{*}$ affects mostly the amplification of the estimated human wrench. Although it is favorable for the effort reduction to set $\boldsymbol{M}_{d}^{*}$ to small values, it cannot be set too low as 
Table II. Inertial parameters of the carried object.

\begin{tabular}{ll}
\hline $\boldsymbol{m}(\mathbf{k g})$ & $\mathbf{1 . 1 4}$ \\
\hline$I_{x}\left(\mathrm{kgm}^{2}\right)$ & 0.0035 \\
$I_{x y}\left(\mathrm{kgm}^{2}\right)$ & 0.0 \\
$I_{x z}\left(\mathrm{kgm}^{2}\right)$ & 0.0 \\
$I_{y}\left(\mathrm{kgm}^{2}\right)$ & 0.0004 \\
$I_{y z}\left(\mathrm{kgm}^{2}\right)$ & 0.0 \\
$I_{z}\left(\mathrm{kgm}^{2}\right)$ & 0.0038 \\
\hline
\end{tabular}

Table III. Control parameters.

\begin{tabular}{ll}
\hline $\boldsymbol{M}_{d}^{*}$ & blockdiag $\left\{9.0 \boldsymbol{I}_{3 \times 3}, 0.4 \boldsymbol{I}_{3 \times 3}\right\}$ \\
$\boldsymbol{K}_{d}$ & blockdiag $\left\{530.0 \boldsymbol{I}_{3 \times 3}, 96.0 \boldsymbol{I}_{3 \times 3}\right\}$ \\
$\boldsymbol{K}_{p}$ & blockdiag $\left\{150.0 \boldsymbol{I}_{3 \times 3}, 25.0 \boldsymbol{I}_{3 \times 3}\right\}$ \\
$\boldsymbol{\Gamma}$ & $\operatorname{diag}\{0.425,0.005,0.005,0.005,0.01,0.01,0.01,0.01,0.01,0.01\}$ \\
$\rho_{j, \infty}$ & $\{0.03,0.03,0.03,0.005,0.005,0.005\}$ \\
$\rho_{j}$ & $\{1,1,1,0.1,0.1,0.1\}$ \\
$s_{j}$ & $\{5,5,5,5,5,5\}$ \\
$k_{j}=$ & $\{1,1,1,1,1,1\}$ \\
\hline
\end{tabular}
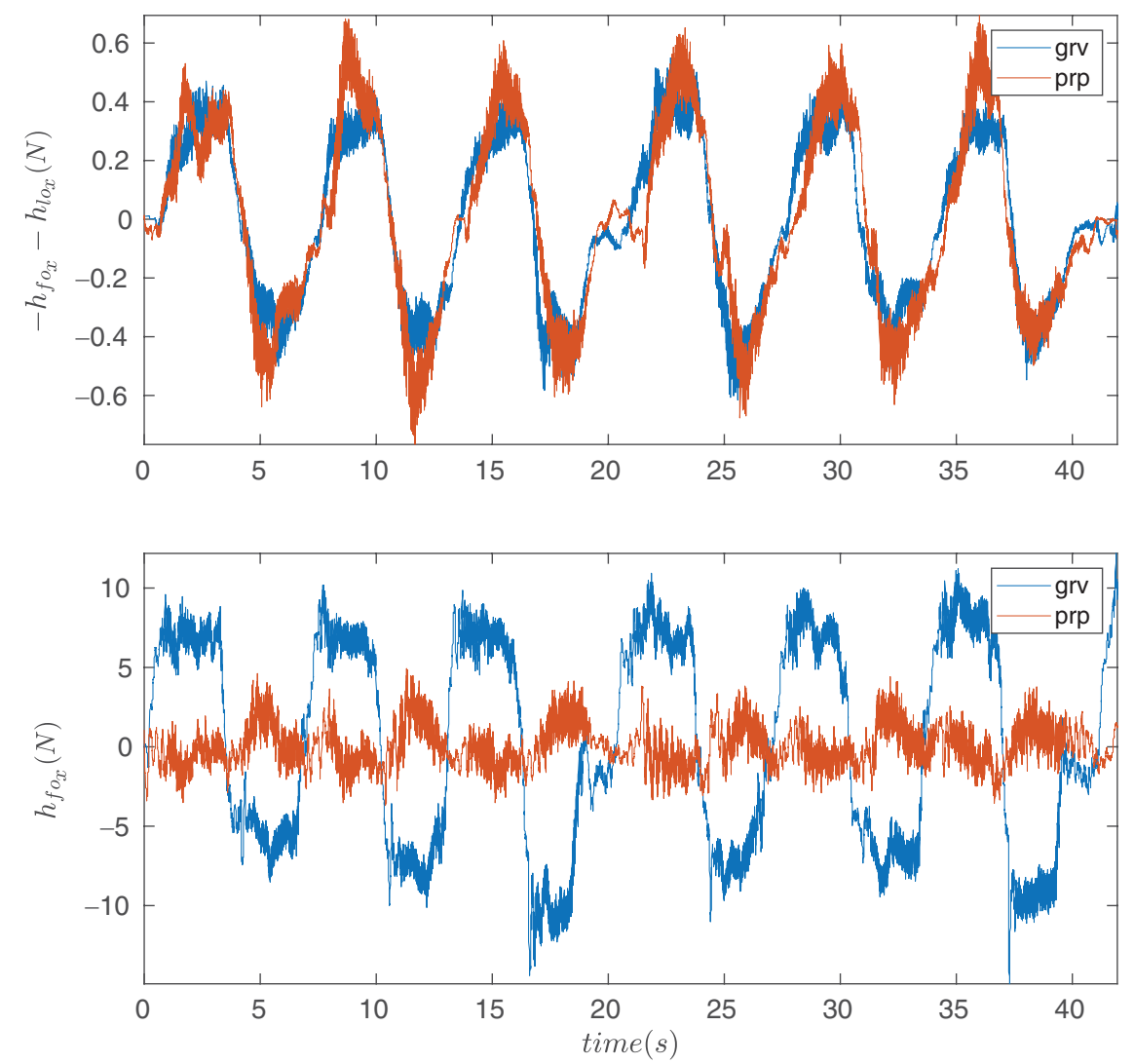

Fig. 5. Force owned to the object's motion and interaction force in $\mathrm{x}$ direction for gravity compensation (grv) and the proposed approach (prp). 

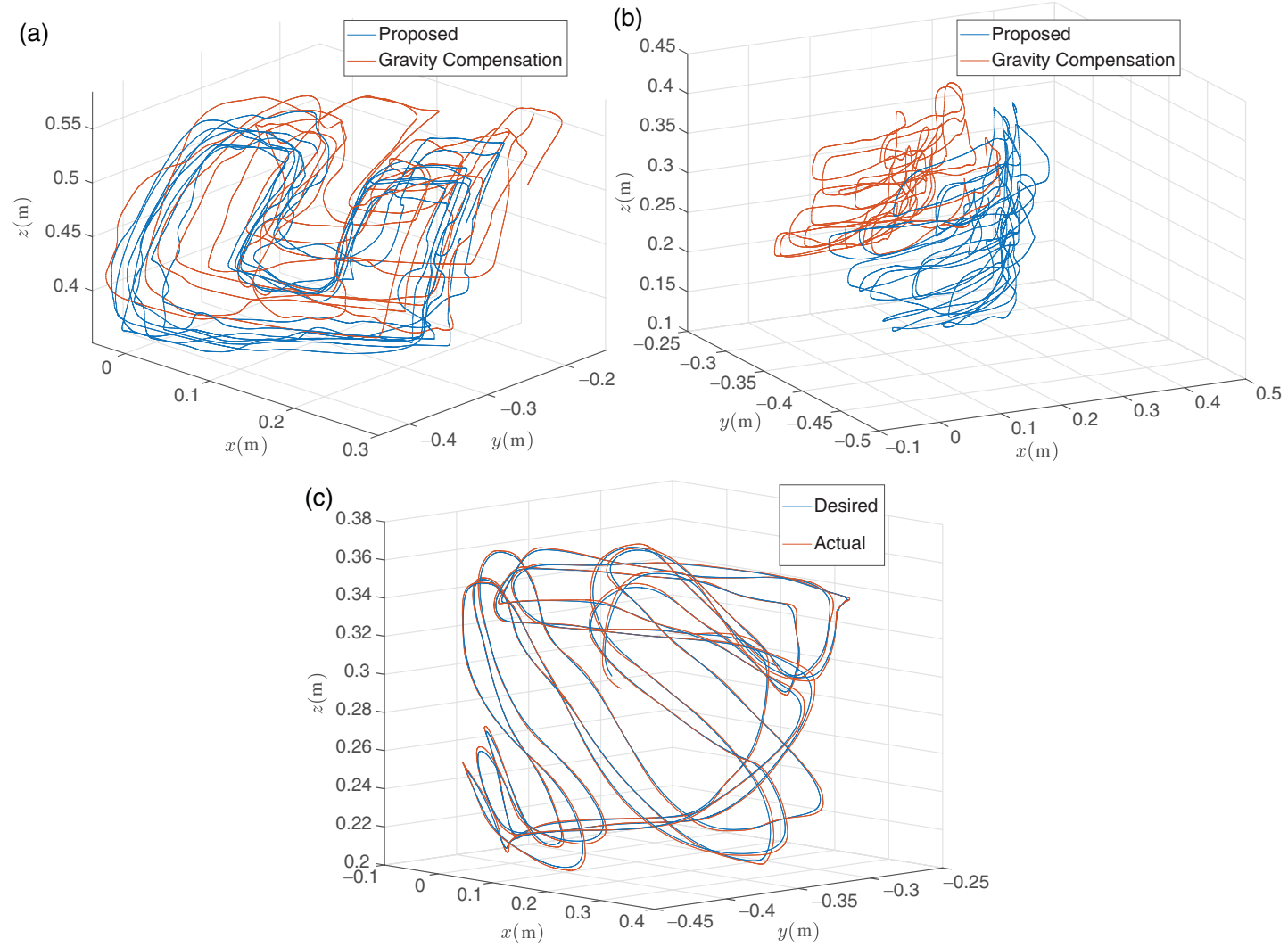

Fig. 6. (a) Accepted trajectories, (b) rejected trajectories and (c) 3D visualization of the estimation law.

sensor noise would be as well amplified. The term $\boldsymbol{M}_{d}^{*-1} \boldsymbol{K}_{d}$ affects damping, and it has been tuned to a rather underdamped behavior that reduces the human effort despite undermining the interaction quality. The stiffness matrix $\boldsymbol{M}_{d}^{*-1} \boldsymbol{K}_{p}$ is tuned high enough to provide stability to the system and low enough to allow sufficient effort reduction. The parameters $k_{j}, s_{j}, \rho_{j}$ involved in ref. [25] are chosen to provide a fast convergence to $\rho_{j, \infty}$. On the other hand, $\rho_{j, \infty}$ is set to values that allow sufficient estimation without the appearance of high-frequency terms in the estimated signal.

As stated above, these experiments aim primarily to quantify the effort reduction of the leader and compare the results with those obtained from gravity compensation operation. For the comparison part, it is instantly indicated that valid data for comparison should be generated, meaning that the compared trajectories should be the same. Thus, the reference tracking operation is employed for this exact reason, that is, as an assistive reference that facilitates the implementation of similar robot trajectories to some extent. However, considering that the reference trajectory imposes a 2D constraint on the $6 \mathrm{DoF}$ manipulator, it is obvious that there are redundancies on the manipulator configurations that can track the reference. These redundancies are undesired as they lead to different trajectories and hinder the comparison. Hence, the subjects were advised to keep the configuration changes between the two methods limited. The experiments that differed significantly based on an empirical inspection were discarded during the procedure. Figure 6 gives an example of valid/accepted and invalid/rejected trajectories. Notice that, although the accepted trajectories are relatively close to each other, they are not identical. In that sense, a suitable index to distinguish those trajectories should be based on the power required by the object to follow this trajectory. Therefore, the RMS value of the power $P_{o}=-\left(\boldsymbol{h}_{f o}+\boldsymbol{h}_{l o}\right)^{T} \boldsymbol{v}_{o}$, that is, $\operatorname{RMS}\left(P_{o}\right)$ is introduced. Similarly, $\operatorname{RMS}\left(P_{f_{o}}\right)$ and $\operatorname{RMS}\left(P_{l o}\right)$ are denoted for the follower and leader case, respectively, where $P_{l o}=\boldsymbol{h}_{l o}^{T} \boldsymbol{v}_{o}, P_{f o}=\boldsymbol{h}_{f o}^{T} \boldsymbol{v}_{o}$. Moreover, we are denoting the RMS value of the $\mathcal{L}_{2}$ norm of force vector as RMS $\left(\left\|f_{k}\right\|_{2}\right)$, and similarly for the torque RMS $\left(\left\|\boldsymbol{\mu}_{k}\right\|_{2}\right)$, where $k \in\{l o, f o, o\}$ and $\left[\boldsymbol{f}_{o}^{T}, \boldsymbol{\mu}_{o}^{T}\right]^{T}=-\left(\boldsymbol{h}_{f o}+\boldsymbol{h}_{l o}\right)$. The aforementioned norms are the main components used on the results of the second set of experiments, and they will be referred to as power, force and torque norms, respectively. The results are presented in Figs. 7 and 8, with rows referring to the subjects. The box plots depict the median and variance of the 

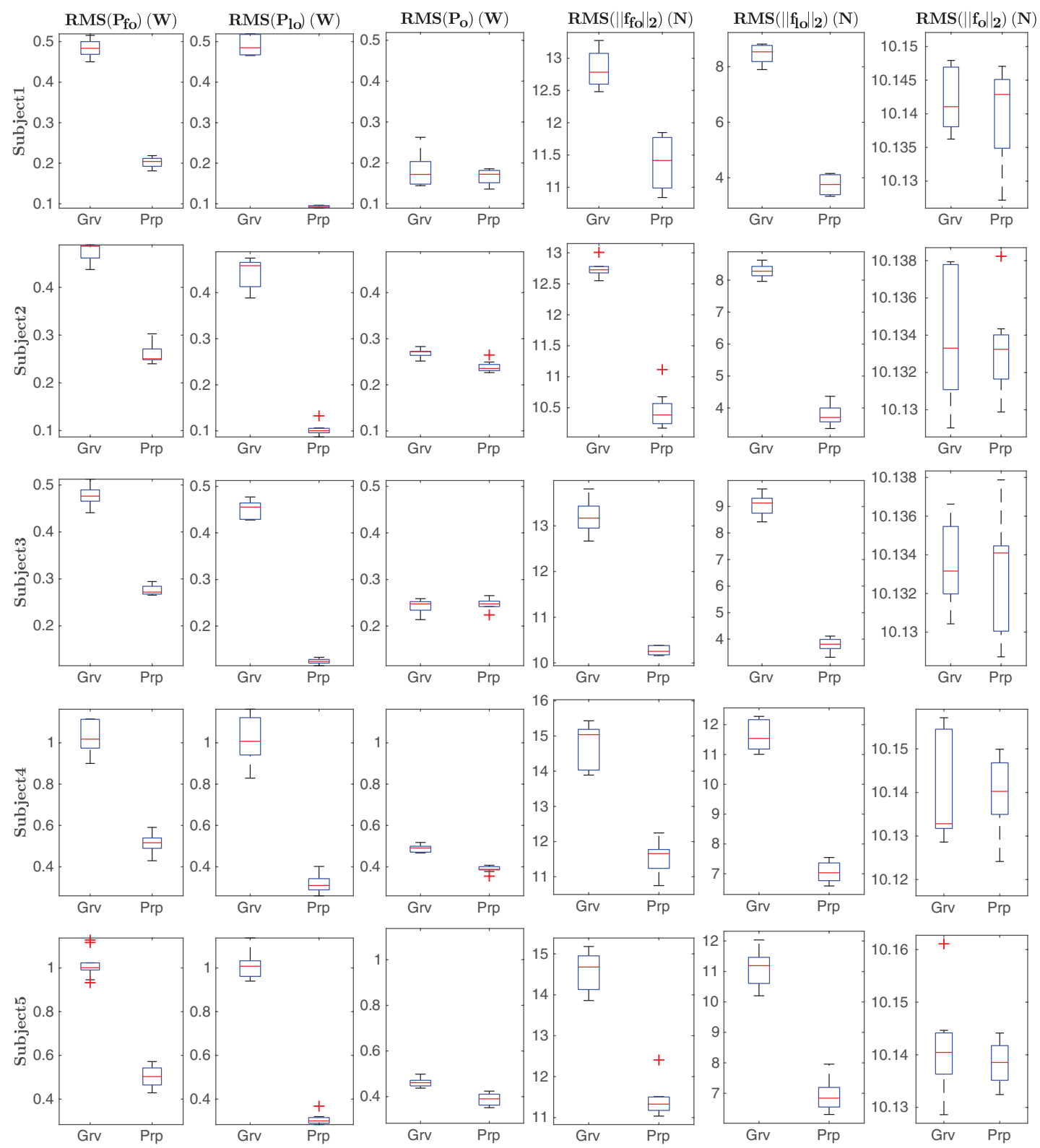

Fig. 7. Box plots of subjects 1-5 regarding the experiment described in Section 4.2.

trajectories that each subject performed for both methods. The first three columns refer to the powerrelated norms introduced above. Notice that in $\operatorname{RMS}\left(P_{o}\right)$ column, the differences between the two methods are comparable, namely the factor $\frac{\operatorname{RMS}\left(P_{\text {oprp }}\right)}{\operatorname{RMS}\left(P_{\text {ogr }}\right)}$ where "prp" stands for proposed approach and "grv" for gravity compensation has a mean value of 0.989 over all subjects with a standard deviation of 0.151 , indicating that the two sets of trajectories are similar also in terms of power. Comparing the two methods, the reduction in $\operatorname{RMS}\left(P_{f}\right)$ is significant, while the reduction in the RMS value of the estimated human power is even higher. As expected, the combination of a successful prediction with an impedance controller limits the interaction forces currently governed by the desired impedance imposed on the system. Notice that unmodeled static friction and damping, which exist in both methods, play a noticeable role in reducing the required human power. The experimental results demonstrate that the proposed controller has shown robustness to the nonlinear terms that derive from the aforementioned unmodeled dynamics. However, in gravity compensation, the human leader 

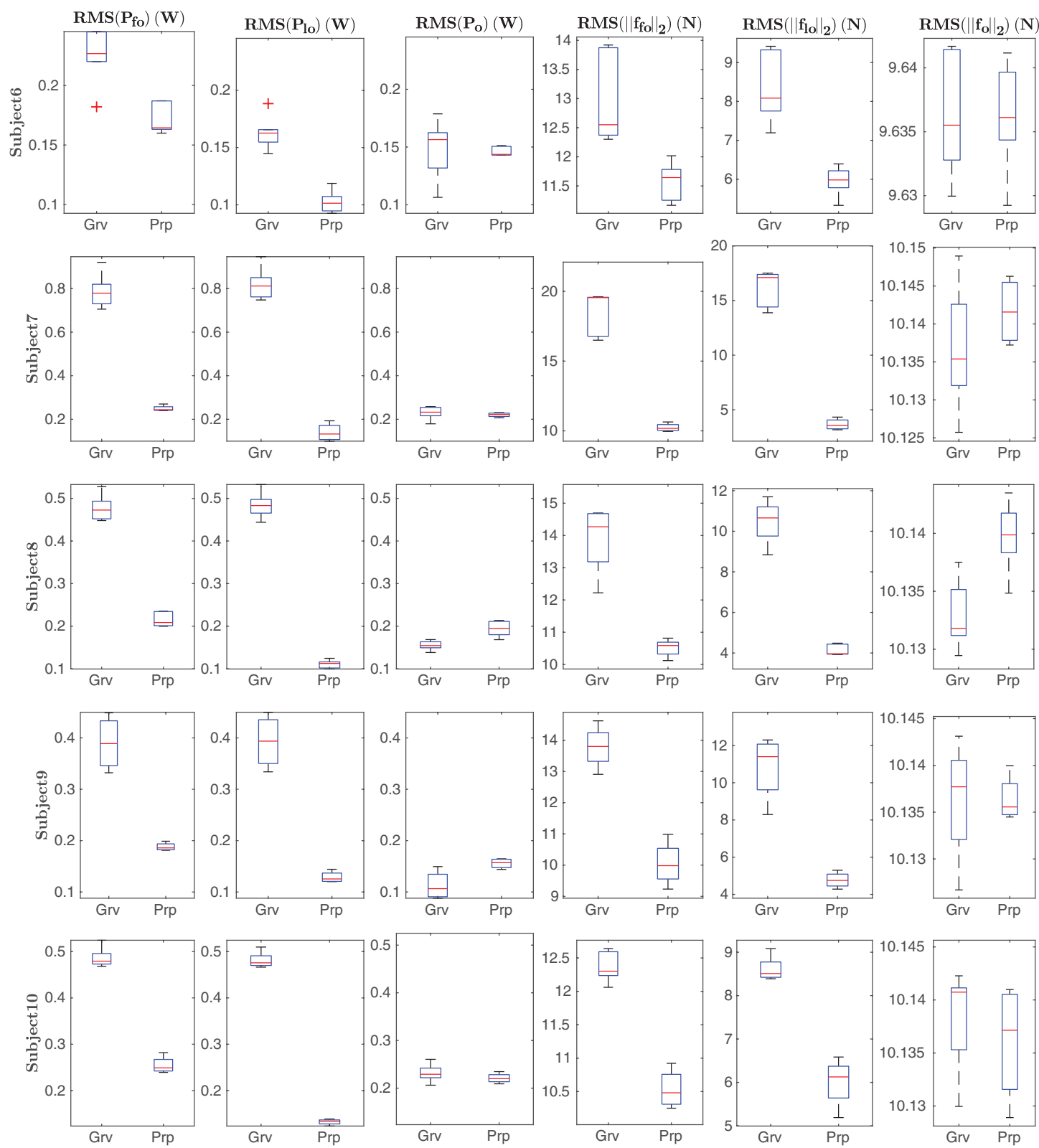

Fig. 8. Box plots of subjects 6-10 regarding the experiment described in Section 4.2.

is responsible for counteracting these forces that is, the human carries both the object and the robot in a zero-gravity environment. Consequently, the increased power requirements on human behalf, compared to $\operatorname{RMS}\left(P_{o}\right)$, are completely justifiable when both quantities refer to gravity compensation. In the same spirit, regarding the proposed approach, the required human power is reduced as compared to $\operatorname{RMS}\left(P_{o}\right)$. Namely, the human can transport the object along a trajectory by providing significantly less power than the required one when the object tracks the exact trajectory on its own. The above result verifies that the manipulator indeed contributes actively to the transportation task, which constitutes the most valuable outcome of this work. In Figs. 7 and 8, the rest of the columns depict the box plots of the force norms, in the same sense as with the power norms described above. These norms may lack physical meaning compared to power, but they are included as a trustworthy indicator. As expected, the force norms support the arguments discussed above. Similar conclusions may be drawn for the corresponding torque norms. 

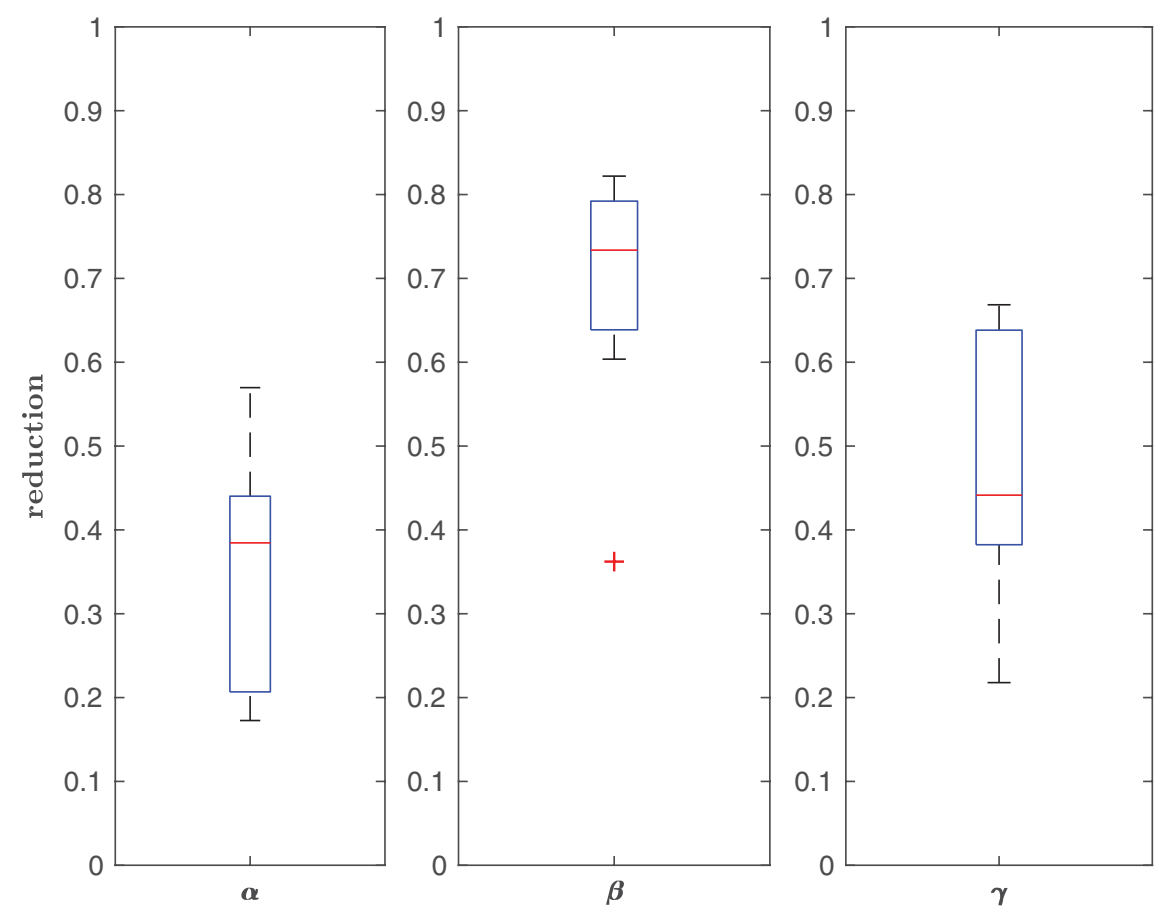

Fig. 9. Box plots of $\alpha, \beta, \gamma$ regarding the experiment described in Section 4.2.

Finally, an attempt to quantify the effort reduction over all subjects has been made. To this end, three new indices will be introduced. Let us denote

$$
\overline{\operatorname{RMS}\left(P_{k_{m}}\right)}=\frac{\sum_{n=1}^{N} \operatorname{RMS}\left(P_{k_{m_{n}}}\right)}{N}
$$

where $k \in\left\{f_{o}, l o, o\right\}, m \in\{$ grv, prp $\}$ stands for the gravity compensation and the proposed approach correspondingly and $n$ indicates a single trajectory of a subject. The three indices are

$$
\begin{aligned}
& \alpha=1-\frac{\overline{\operatorname{RMS}\left(P_{l_{p r p}}\right)}}{\overline{\operatorname{RMS}\left(P_{o_{p r p}}\right)}} \\
& \beta=1-\frac{\overline{\operatorname{RMS}\left(P_{l_{p r p}}\right)}}{\overline{\operatorname{RMS}\left(P_{l_{g r v}}\right)}} \\
& \gamma=1-\frac{\overline{\operatorname{RMS}\left(P_{f_{p r p}}\right)}}{\overline{\operatorname{RMS}\left(P_{f_{g r v}}\right)}}
\end{aligned}
$$

Notice that before computing the indices, the values $\overline{\operatorname{RMS}\left(P_{l_{g r v}}\right)}$ and $\overline{\operatorname{RMS}\left(P_{f_{g r v}}\right)}$ were scaled by the factor $\frac{\overline{\operatorname{RMS}\left(P_{\left.o_{p r p}\right)}\right)}}{\overline{\operatorname{RMS}\left(P_{o_{g r v}}\right)}}$ in order to convey a fair comparison. The box plots for the above indices over the subjects are presented in Fig. 9. A mean reduction of $35.67 \%$ with a standard deviation of 0.13 is revealed in the required human power during the transportation task. Moreover, the mean reduction of the required human power in our approach as compared to the required human power in gravity compensation is $69.78 \%$ with a standard deviation of 0.13 . Finally, the mean reduction of power owned to the interaction forces in our approach compared to the corresponding power in gravity compensation is $47.58 \%$ with a standard deviation of 0.14 .

\subsection{Unknown object dynamics}

A similar set of experiments with the second set presented in Section 4.2 was designed to show the efficacy of the adaptive law. In this set, we used the same object (and laser) but we modeled it as 

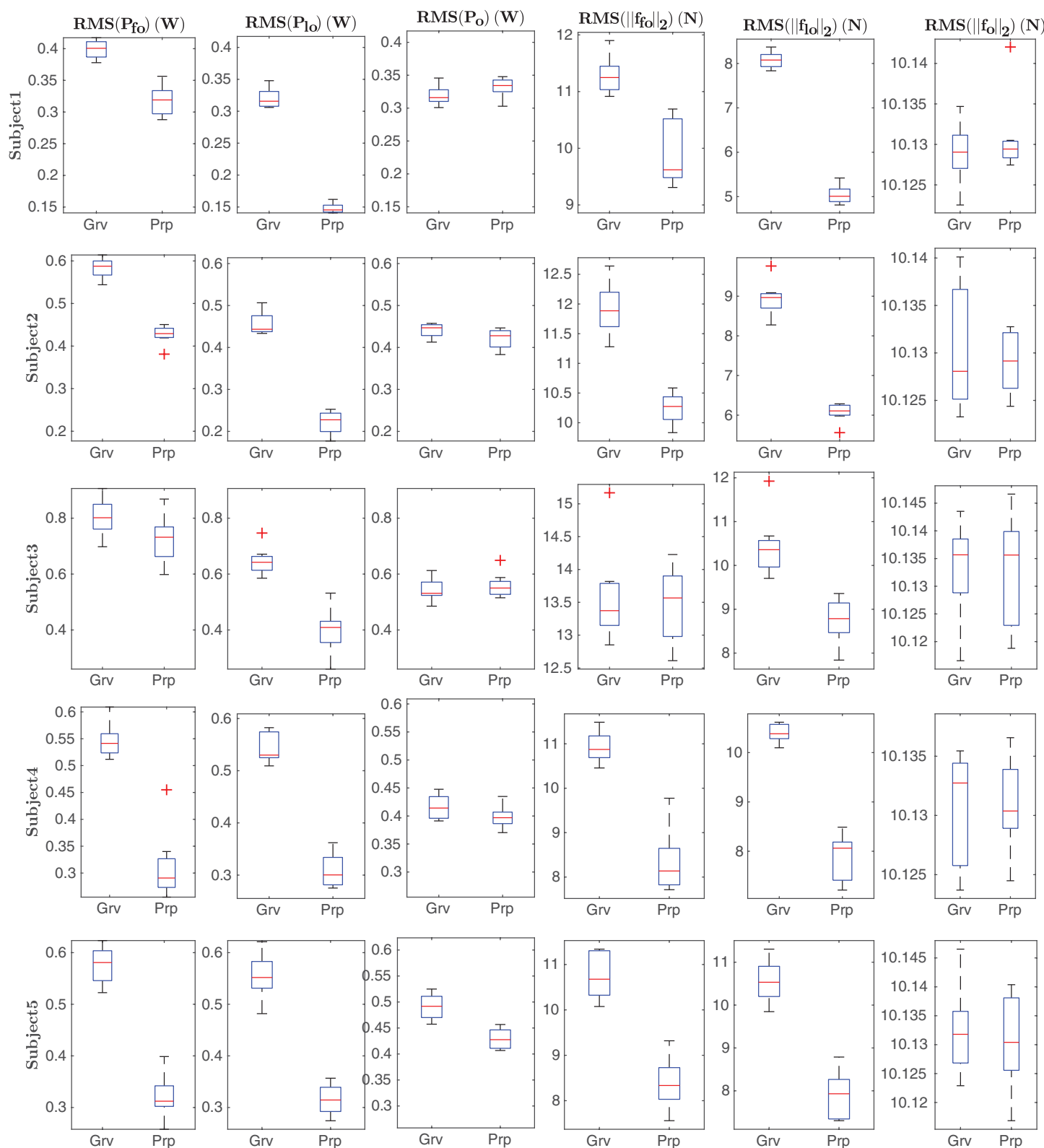

Fig. 10. Box plots of subjects 1-5 regarding the experiment described in Section 4.3.

a point mass in both methods. Moreover, the point mass is just a fraction of the object's real mass. This fraction spanned from $0.5 \%$ to $0.7 \%$ (with mean $0.58 \%$ ). Only subjects $1-5$ participated in this experiment. Each subject performed 9 trials for each method for only one reference trajectory. To enhance excitation, the trajectory was chosen to be substantially richer in content than the previous trajectories. Every other part of the experiment was the same as the previous experiment. The results are depicted in Fig. 10, where the same analysis with Section 4.2 holds. The indices introduced in Section 4.2 have been employed here as well. The results in Fig. 10 reveal a mean reduction of $49.4 \%$ with a standard deviation of 0.16 on index $\alpha$. For indices $\beta, \gamma$, the mean reduction is $59.72 \%$ and $27.28 \%$ with standard deviations of 0.08 and 0.11 , respectively. Although $\beta, \gamma$ reductions verify the concept of this experiment, a comparison with the results of Fig. 11 is discouraged due to the high bias that comes from using the same trajectory over all experiments. However, the results on $\alpha$ make a more valid point as they are less affected by the choice of the trajectory. It should be noted that fine-tuning of the gains took place initially, but on the other hand, the adaptive law combined with a $\sigma$-modification term dealt with a rough initial estimation of object parameters and added robustness 

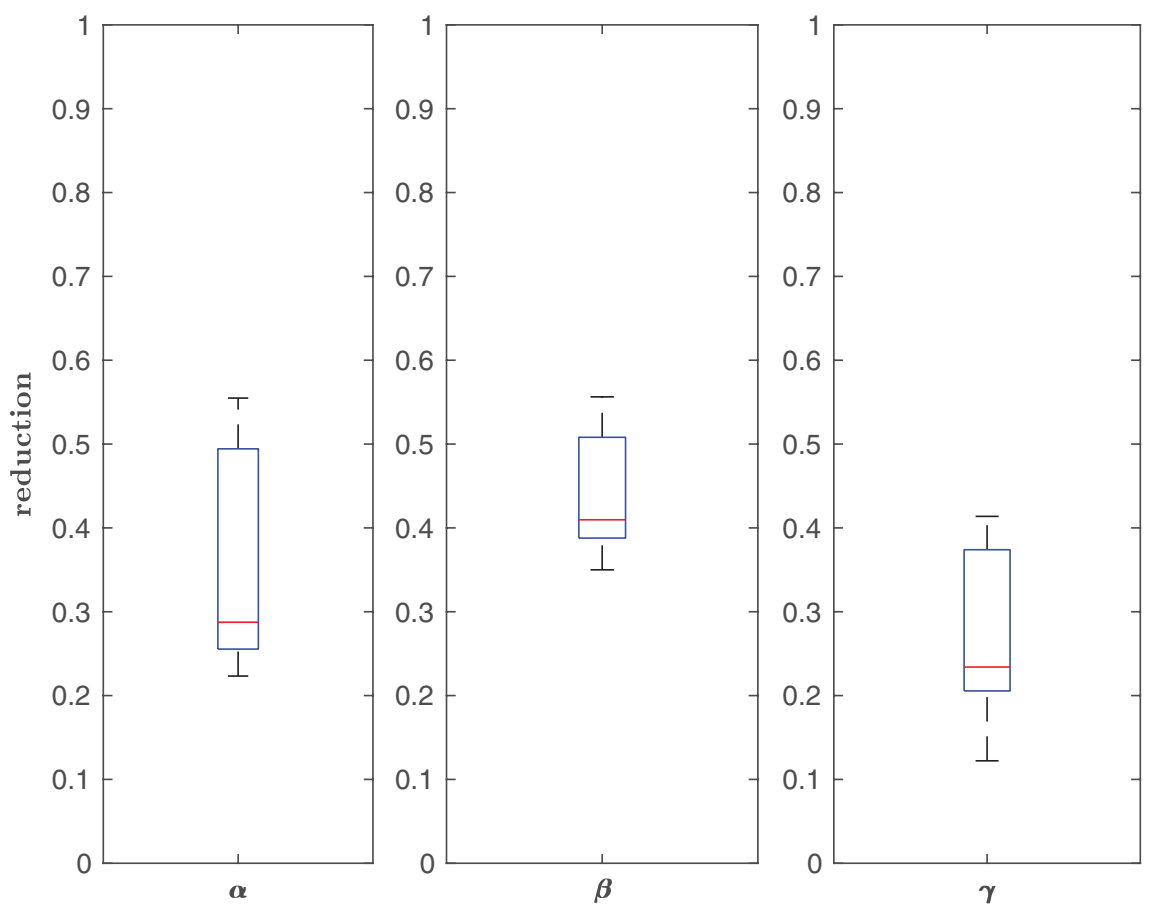

Fig. 11. Box plots of $\alpha, \beta, \gamma$ regarding the experiment described in Section 4.3.
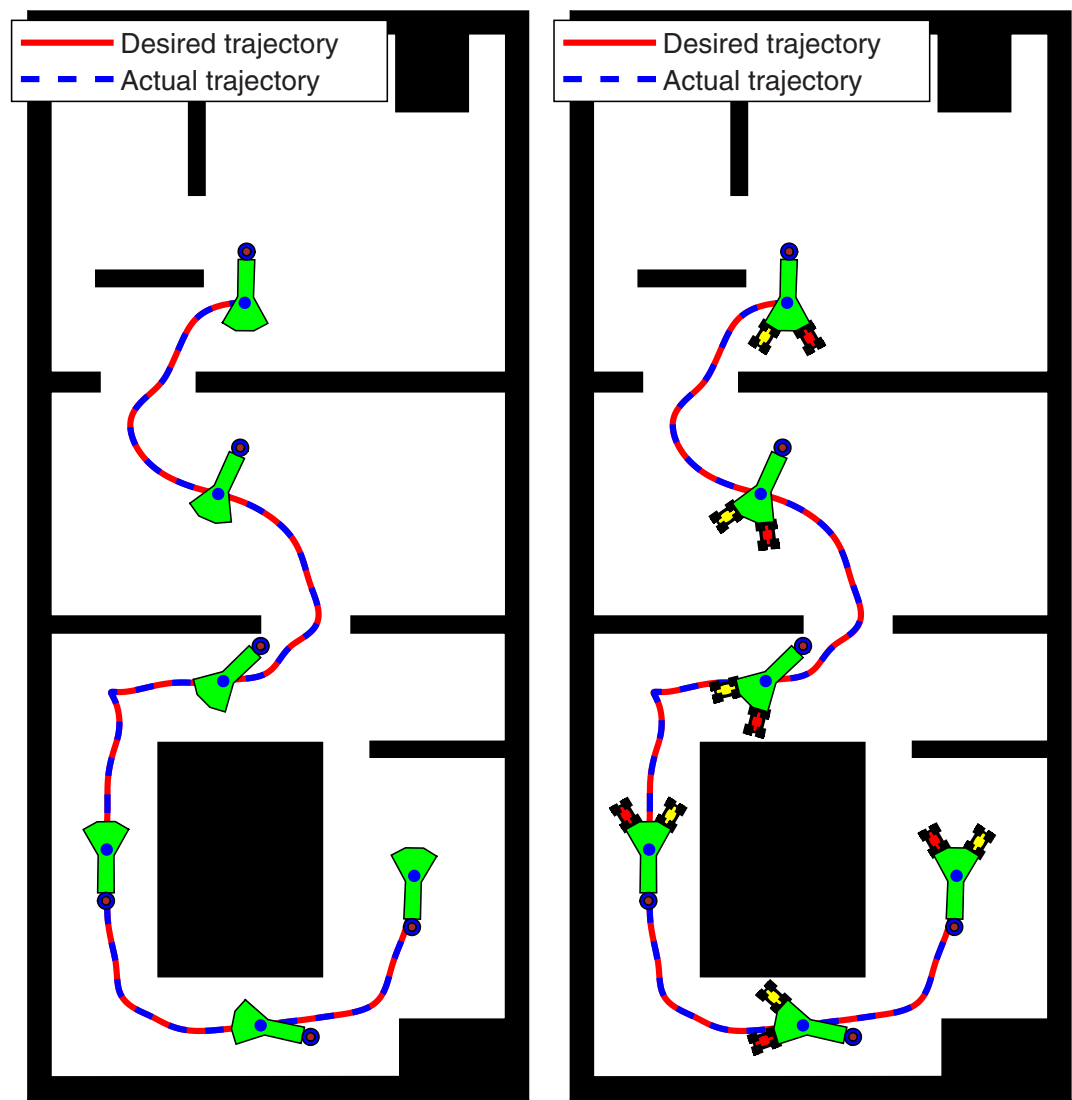

Fig. 12. Simulation results indicating the desired trajectory tracking during (a) human (blue circle with red center) moving the object (green) alone and (b) the proposed cooperative scheme (red and yellow robots). In both cases, the tracking is satisfactory (i.e., (a) $\operatorname{RMS}\left(\left\|\boldsymbol{e}_{p}\right\|_{2}\right)=0.0085 \mathrm{~m}, \operatorname{RMS}(\theta)=0.011 \mathrm{rad}$, (b) $\operatorname{RMS}\left(\left\|\boldsymbol{e}_{p}\right\|_{2}\right)=$ $0.0089 \mathrm{~m}, \operatorname{RMS}(\theta)=0.012 \mathrm{rad}$, where $\theta$ is the orientation.) 
(a)
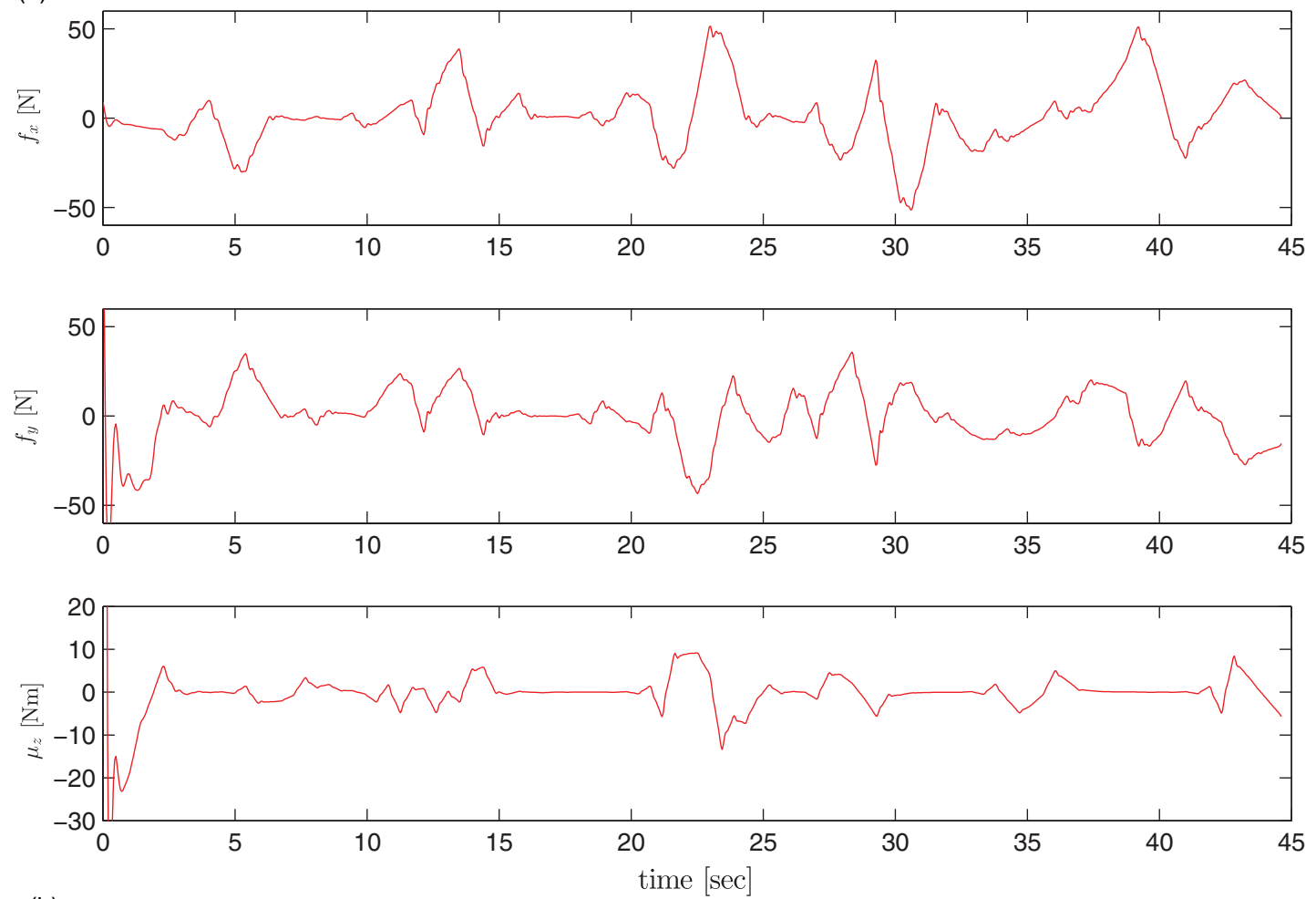

(b)
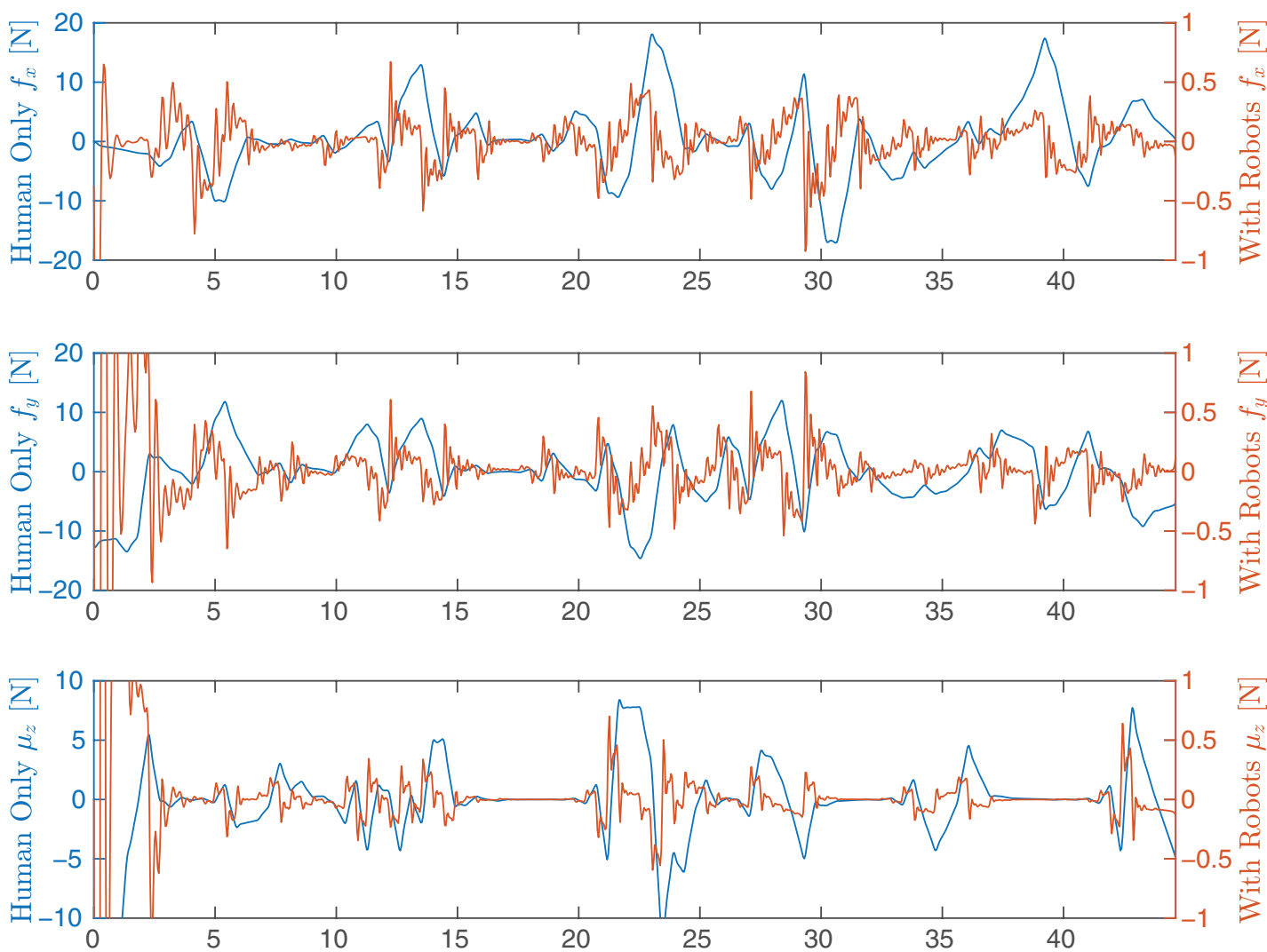

Fig. 13. Simulation results depicting (a) forces/torque exerted on the object's center by the robots, (b) the human effort both when human operates alone and when cooperates with the robots. 
to the system. Nonetheless, even though it may assist in the collaboration tasks, there is no guarantee of convergence to the actual parametric values owing to poor excitation (Chapter 4 in ref. [30]).

\section{Simulation Results}

To verify the proposed algorithm for multiple robotic agents, simulations for a planar and oriented motion were developed. Specifically, two holonomic mobile robots rigidly grasped an object along with the human user to fulfill a complex trajectory in a confined workspace. In the simulation, a collision-free trajectory was given as reference to the human leader, which was modeled as another robotic manipulator operating under a PID control law, while the wrench exerted from each robot's end effector to the object was considered globally known. The force and torque measurements actually involved gaussian noise with zero mean value and 5\% deviation of the actual value as well as delay of $1 \mathrm{~ms}$. For comparison purposes, the same desired trajectory was used to simulate the transportation task, implemented exclusively by the human, in order to quantify the collaborative gain when the robots are employed. The parameters used in the simulation were $m_{o}=10 \mathrm{~kg}, \boldsymbol{I}_{o}=6 \mathrm{~kg} \mathrm{~m}^{2}$, $\boldsymbol{M}_{f_{1,2}}=\operatorname{blockdiag}\left\{20 \boldsymbol{I}_{2 \times 2}, 1\right\}, \boldsymbol{M}_{d}=\boldsymbol{I}_{3 \times 3}, \boldsymbol{K}_{d}=2 \boldsymbol{I}_{3 \times 3}, \boldsymbol{K}_{p}=\boldsymbol{I}_{3 \times 3}, \rho_{j, \infty}=0.1, \rho_{j}=1, s_{j}=0.5, k_{j}=4$. As shown in Fig. 12 the tracking ability of the overall collaborative scheme is comparable to the tracking with the human alone. Moreover, contrasting the human interaction forces/torque in Fig. 13, a large reduction (about 20 times on forces and 10 times on torque) is deduced. Notice that the figure is suitably scaled for viewing purposes. The behavior of the first 3 seconds occurs due to the convergence of the estimation law. Even with a bad initial estimation though, the forces/torques do not exceed the ones produced in the human-only case. Although, the simulation results validate the proposed algorithm, the corresponding reduction in a real application would be lower due to hardware limitations and especially the $\mathrm{f} / \mathrm{t}$ sensor's characteristics. Specifically, noisy and inaccurate measurements would deteriorate the overall performance and would lead to a more conservative tuning policy that would penalize the effort reduction.

\section{Conclusion}

In this work, an efficient framework was developed to deal with the emerging field of cooperative object manipulation through pHRI. A prescribed performance estimator was successfully employed to robustly estimate complex trajectories determined by the human leader. The impedance characteristics combined with the motion intention estimation led to significant human effort reduction. Moreover, the robot's position, velocity and force/torque measurements at its end effector were the only online sensing needed to implement the control scheme. Furthermore, the exact knowledge of the object dynamics was relaxed via an appropriately designed adaptive scheme. Multiple robotic coworkers were considered in order to cover transportation tasks involving heavy and bulky objects. Integration of collision avoidance in the proposed approach along with human wrench estimation without explicit communication in the multiple robotic coworkers case are left open for future research.

\section{Funding}

This work was supported by the EU funded project Co4Robots: Achieving Complex Collaborative Missions via Decentralized Control and Coordination of Interacting Robots (H2020-ICT-731869, 2017-2019) and the Hellenic Foundation for Research and Innovation (H.F.R.I.) under the First Call for H.F.R.I. Research Projects to support Faculty members and Researchers and the procurement of high-cost research equipment grant (Project Number: 466).

\section{References}

1. Y. Hirata, Y. Kume, Z. Wang and K. Kosuge, "Decentralized Control of Multiple Mobile Manipulators Based on Virtual 3-D Caster Motion for Handling an Object in Cooperation with a Human," Proceedings IEEE International Conference on Robotics and Automation, vol. 1 (2003) pp. 938-943.

2. Y. Hirata and K. Kosuge, "Distributed Robot Helpers Handling a Single Object in Cooperation with a Human," Proceedings - IEEE International Conference on Robotics and Automation, vol. 1 (2000) pp. 458-463.

3. A. Mörtl, M. Lawitzky, A. Kucukyilmaz, M. Sezgin, C. Basdogan and S. Hirche, "The role of roles: Physical cooperation between humans and robots," Int. J. Robot. Res. 31(13), 1656-1674 (2012). 
4. B. Corteville, E. Aertbelien, H. Bruyninckx, J. De Schutter and H. Van Brussel, "Human-Inspired Robot Assistant for Fast Point-to-Point Movements," Proceedings - IEEE International Conference on Robotics and Automation (2007) pp. 3639-3644.

5. Y. Maeda, T. Hara and T. Arai, "Human-Robot Cooperative Manipulation with Motion Estimation," Proceedings 2001 IEEE/RSJ International Conference on Intelligent Robots and Systems. Expanding the Societal Role of Robotics in the the Next Millennium (Cat. No. O1CH37180), vol. 4 (IEEE, 2001) pp. 2240-2245.

6. A. Thobbi, Y. Gu and W. Sheng, "Using Human Motion Estimation for Human-Robot Cooperative Manipulation," In: 2011 IEEE/RSJ International Conference on Intelligent Robots and Systems (IEEE, 2011) pp. 2873-2878.

7. L. Roveda, "A User-Intention Based Adaptive Manual Guidance with Force-Tracking Capabilities Applied to Walk-Through Programming for Industrial Robots," In: 2018 15th International Conference on Ubiquitous Robots (UR) (2018).

8. L. Roveda, S. Haghshenas, M. Caimmi, N. Pedrocchi and L. Molinati Tosatti, "Assisting operators in heavy industrial tasks: On the design of an optimized cooperative impedance fuzzy-controller with embedded safety rules," Front. Robot. AI 6, 75 (2019).

9. L. Roveda, J. Maskani, P. Franceschi, A. Abdi, F. Braghin, L. M. Tosatti and N. Pedrocchi, "Model-based reinforcement learning variable impedance control for human-robot collaboration," J. Intell. Robot. Syst., 1-17 (2020).

10. Y. Li and S. S. Ge, "Human-robot collaboration based on motion intention estimation," IEEE/ASME Trans. Mechatron. 19(3), 1007-1014 (2014).

11. P. Evrard, E. Gribovskaya, S. Calinon, A. Billard and A. Kheddar, "Teaching Physical Collaborative Tasks: Object-Lifting Case Study with a Humanoid," In: 9th IEEE-RAS International Conference on Humanoid Robots, HUMANOIDS09 (2009) pp. 399-404.

12. L. Rozo, D. Bruno, S. Calinon and D. G. Caldwell, "Learning Optimal Controllers in Human-Robot Cooperative Transportation Tasks with Position and Force Constraints," In: IEEE International Conference on Intelligent Robots and Systems, vol. 2015-December (2015) pp. 1024-1030.

13. J. R. Medina, M. Lawitzky, A. Mörtl, D. Lee and S. Hirche, "An Experience-Driven Robotic Assistant Acquiring Human Knowledge to Improve Haptic Cooperation," In: IEEE International Conference on Intelligent Robots and Systems (2011) pp. 2416-2422.

14. M. Uchiyama and P. Dauchez, "A Symmetric Hybrid Position/Force Control Scheme for the Coordination of Two Robots," Proceedings of the 1988 IEEE International Conference on Robotics and Automation (IEEE, 1988) pp. 350-356.

15. O. Khatib, "Object Manipulation in a Multi-Effector Robot System," Proceedings of the 4th International Symposium on Robotics Research, vol. 4 (MIT Press, 1988) pp. 137-144.

16. M. M. Rahman, R. Ikeura and K. Mizutani, "Investigation of the impedance characteristic of human arm for development of robots to cooperate with humans," JSME Int. J. Ser. C: Mech. Syst. Mach. Elem. Manuf. 45(2), 510-518 (2002).

17. I. D. Walker, R. A. Freeman and S. I. Marcus, "Analysis of motion and internal loading of objects grasped by multiple cooperating manipulators," Int. J. Robot Res. 10(4), 396-409 (1991).

18. J. Hollerbach, W. Khalil and M. Gautier, "Model Identification," In: Springer Handbook of Robotics (Springer, 2008) pp. 321-344.

19. C. P. Bechlioulis and G. A. Rovithakis, "Robust partial-state feedback prescribed performance control of cascade systems with unknown nonlinearities," IEEE Trans. Automat. Cont. 56(9), 2224-2230 (2011).

20. E. D. Sontag, Mathematical Control Theory (Springer, London, UK, 1998).

21. K. Kosuge and T. Oosumi, "Decentralized Control of Multiple Robots Handling an Object," Proceedings of the IEEE International Conference on Intelligent Robots and Systems (vol. 1, 1996) pp. 318-323.

22. K. Kosuge, T. Oosumi and K. Chiba, "Load Sharing of Decentralized-Controlled Multiple Mobile Robots Handling a Single Object," Proceedings of the IEEE International Conference on Robotics and Automation (vol. 4, 1997) pp. 3373-3378.

23. K. Kosuge, T. Oosumi and H. Seki, "Decentralized Control of Multiple Manipulators Handling an Object in Coordination Based on Impedance Control of Each Arm," Proceedings of the IEEE International Conference on Intelligent Robots and Systems (vol. 1, 1997) pp. 17-22.

24. B. Siciliano and L. Villani, Robot Force Control, vol. 540 (Springer Science \& Business Media, 2012).

25. L. Villani and J. De Schutter, "Force Control," In: Springer Handbook of Robotics (Springer, 2008) pp. 161-185.

26. F. Caccavale, B. Siciliano and L. Villani, "Quaternion-Based Impedance with Nondiagonal Stiffness for Robot Manipulators," Proceedings of the 1998 American Control Conference, vol. 1. (IEEE, 1998) pp. 468-472.

27. Z. Wang and M. Schwager, "Multi-Robot Manipulation Without Communication," In: Distributed Autonomous Robotic Systems (Springer, 2016) pp. 135-149.

28. P. Hsu, "Coordinated control of multiple manipulator systems," IEEE Trans. Robot. Automat. 9(4), 400-410 (1993).

29. C. Mavridis, K. Alevizos, C. P. Bechlioulis and K. J. Kyriakopoulos, "Human-Robot Collaboration Based on Robust Motion Intention Estimation with Prescribed Performance," In: 2018 European Control Conference, Limassol, Cyprus, vol. 1 (2018) pp. 249-254.

30. P. A. Ioannou and J. Sun, Robust Adaptive Control (Courier Corporation, 2012). 